\title{
Uniting C1-Ammonium Enolates and Transition Metal Electrophiles via Cooperative Catalysis: The Direct, Asymmetric $\alpha$-Allylation of Aryl Acetic Acid Esters.
}

\author{
Kevin J. Schwarz, Jessica L. Amos, J. Cullen Klein, Dung T. Do; Thomas N. Snaddon* \\ Department of Chemistry, Indiana University, 800 E. Kirkwood Ave., Bloomington, Indiana 47405, United \\ States. \\ Corresponding Author: tsnaddon@indiana.edu
}

$\begin{array}{lr}\text { General Information } & \text { S2 }\end{array}$

Experimental Section $\quad$ S3

Catalysts $\quad$ S3

Preparation of esters - Table $1 \quad$ S3

Preparation of allyl partners - Table $1 \quad$ S5

Assessment of selected amine catalysts $\quad$ S7

Assessment of selected leaving groups $\quad$ S8

Preparation of esters - Table $2 \quad$ S9

Preparation of cinnamyl tert-butylcarbonates - Table $2 \quad \mathbf{S 1 3}$

Preparation of product esters - Table $2 \quad \mathbf{S 1 5}$

Large-scale preparations of 6 and $18 \quad$ S26

Transformations of $\mathbf{6}$ (Scheme 2) and confirmation $\quad \mathbf{S 2 7}$

of absolute stereochemistry

Transformations of $\mathbf{6}$ - HPLC chromatograms $\quad$ S29

$\begin{array}{ll}\text { References } & \text { S35 }\end{array}$ 


\section{General Information}

Commercial reagents were purified prior to use following the guidelines of Perrin and Armarego. ${ }^{1}$ Unless otherwise noted, all reactions have been carried out with distilled and degassed solvents under an atmosphere of dry $\mathrm{N}_{2}$ in flame or oven dried glassware with standard vacuum-line techniques. All reactions were carried out in Teflon screw cap reaction vials with magnetic stirring unless otherwise indicated. Dichloromethane and tetrahydrofuran were purified under a positive pressure of dry argon by passage through two columns of activated alumina. Toluene was purified under a positive pressure of dry argon by passage through columns of activated alumina and Q5 (Grubbs apparatus). All workup and purification procedures were carried out with reagent grade solvents (purchased from Sigma-Aldrich) in air. Standard column chromatography techniques using ZEOprep 60/40-63 $\mu \mathrm{m}$ silica gel were used for purification. Liquids and solutions were transferred via syringe or cannula.

${ }^{1} \mathrm{H}$ and ${ }^{13} \mathrm{C}$ NMR spectra were recorded at room temperature on Varian Inova-instrumentation: Varian I400 $\left({ }^{1} \mathrm{H}\right.$ NMR at $400 \mathrm{MHz}$ and ${ }^{13} \mathrm{C}$ NMR at $\left.100 \mathrm{MHz}\right)$, Varian VXR400 $\left({ }^{1} \mathrm{H}\right.$ NMR at 400 $\mathrm{MHz}$ and ${ }^{13} \mathrm{C}$ NMR at $\left.100 \mathrm{MHz}\right)$, and Varian I500 $\left({ }^{1} \mathrm{H} \mathrm{NMR}\right.$ at $500 \mathrm{MHz}$ and ${ }^{13} \mathrm{C}$ NMR at 125 $\mathrm{MHz}$ ) using deuterium lock. Data for ${ }^{1} \mathrm{H}$ NMR spectra are quoted relative to chloroform or benzene as an internal standard $(7.26 \mathrm{ppm}$ or $2.1 \mathrm{ppm})$ and data for ${ }^{13} \mathrm{C}$ NMR spectra are quoted relative to chloroform or benzene as an internal standard (77.23 ppm or $128.39 \mathrm{ppm})$ and are reported in terms of chemical shift $(\delta \mathrm{ppm})$. Data are reported as follows: chemical shift, integration, multiplicity $(\mathrm{s}=$ singlet, $\mathrm{d}=$ doublet, $\mathrm{t}=$ triplet, $\mathrm{q}=$ quartet, $\mathrm{br}=$ broad, $\mathrm{m}=$ multiplet), and coupling constants (Hz). Infrared spectra (IR) were obtained on an Avatar 360-FT IR E.S.P. on a NaCl salt plate and recorded in wavenumbers $\left(\mathrm{cm}^{-1}\right)$. Bands are characterized as broad (br), strong (s), medium (m), and weak (w). Melting points were obtained on a Thomas Hoover capillary melting point apparatus without correction. High Resolution Mass (HRMS) analysis was obtained using Electron Impact Ionization (EI) or Chemical Ionization (CI) and reported as $\mathrm{m} / \mathrm{z}$ (relative intensity) for the $[\mathrm{M}]^{+}$or $[\mathrm{M}+\mathrm{H}]^{+}$molecular ion. GC-MS data was acquired using an Agilent 6890N Gas Chromatograph and 5973 Inert Mass Selective Detector. Chiral HPLC analyses were performed on an Agilent 1200 Series system. 


\section{Experimental Section:}

Preparation of starting materials.

\section{Catalysts:}

$(R)-(+)$-Benzotetramisole [(+)-BTM, CAS: 885051-07-0] and $(S)-(-)$-Benzotetramisole [(-)BTM, CAS: 950194-37-3] were purchased from TCI America and used as received, or prepared from (R)-(-)-2-phenylglycinol or $(\mathrm{S})-(+)-2-$ phenylglycinol, respectively, by the procedure of Smith and co-workers.

The $3^{\text {rd }}$ Generation XantphosPd precatalyst was prepared by the procedure of Buchwald and coworkers. $^{3}$

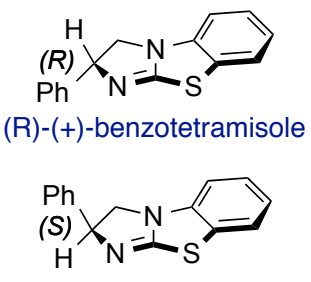

(S)-(-)-benzotetramisole

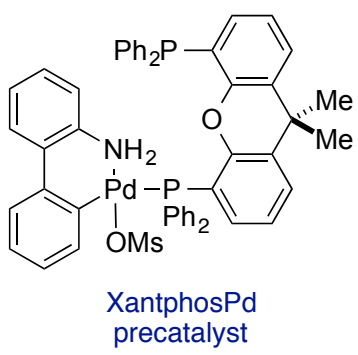

XantphosPd

\section{Preparation of Esters - Table 1.}

\section{General Procedure:}

The specified phenylacetic acid (1.65 mmol, 1.0 equiv.), the specified phenol $(0.25 \mathrm{~g}, 1.82$ mmol, 1.1 equiv.) and dry $\mathrm{CH}_{2} \mathrm{Cl}_{2}(40 \mathrm{~mL})$ were added sequentially to a dry round-bottom flask at room temperature under an atmosphere of nitrogen. The reaction was cooled to $0{ }^{\circ} \mathrm{C}$ using an ice bath and DCC (0.37 g, $1.82 \mathrm{mmol}, 1.1$ equiv.) and DMAP (20 mg, $0.165 \mathrm{mmol}, 0.1$ equiv.) added sequentially. The reaction was allowed to slowly warm to room temperature and further stirred for 16 hours. Thereafter, aq. $\mathrm{HCl}(3 N, 2.0 \mathrm{~mL})$ was added and the reaction placed in a freezer $\left(-20^{\circ} \mathrm{C}\right)$ for a minimum of $6 \mathrm{~h}$ The resulting suspension was filtered over celite and the residue washed with ice-cold $\mathrm{CH}_{2} \mathrm{Cl}_{2}$. The combined filtrates were successively washed with sat. aq. $\mathrm{NaHCO}_{3}$ and water before being dried $\left(\mathrm{MgSO}_{4}\right)$ and concentrated under reduced pressure. The residue was purified by column chromatography $\left[\mathrm{SiO}_{2}\right.$, specified eluent].

4-Nitrophenyl 2-phenylacetate (1): Prepared according to the General Procedure. The title $\mathrm{O}^{\mathrm{NO}_{2}}$ compounds was obtained $(226 \mathrm{mg}, 0.88 \mathrm{mmol}, 53 \%)$ as a white solid following purification by column chromatography $\left[\mathrm{SiO}_{2}\right.$, Petroleum Ether-EtOAc, 5:1].

Spectroscopic data was consistent with the literature report. ${ }^{4}$ 
2,4,6-Trichlorophenyl 2-phenylacetate (2): Prepared according to the General Procedure. The EtOAc, 5:1].
flition by column chromatography [SiO 2 , Petroleum EtherIR (film): 1776, 1447, 1104, 910, $727 \mathrm{~cm}^{-1}$.

${ }^{1} \mathrm{H}$ NMR (400 MHz, $\left.\mathrm{CDCl}_{3}\right): \delta=7.37-7.20(\mathrm{~m}, 7 \mathrm{H}), 3.89(\mathrm{~s}, 2 \mathrm{H})$

${ }^{13} \mathrm{C}$ NMR $\left(101 \mathrm{MHz}, \mathrm{CDCl}_{3}\right): \delta=167.77,146.86,129.51,128.66,128.51,128.04,127.55$, $125.31,121.55,40.53$.

HRMS (EI): $m / z$ calc. for $[\mathrm{M}]^{+} \mathrm{C}_{14} \mathrm{H}_{9} \mathrm{Cl}_{3} \mathrm{O}_{2}:$ 313.97. Found: 313.97 .

2,3,5,6-Tetraflourophenyl 2-phenylacetate (3): Prepared according to the General Procedure. The title compounds was obtained $(236 \mathrm{mg}, 0.85 \mathrm{mmol}, 50 \%)$ as a white
solid following purification by column chromatography $\left[\mathrm{SiO}_{2}\right.$, Petroleum
Ether-EtOAc, 5:1].

M.p. (pentane-EtOAc, $400: 1$; needles) $=45-46{ }^{\circ} \mathrm{C}$

IR (film): 1792, 1525, 1488, $1098 \mathrm{~cm}^{-1}$.

${ }^{1} \mathrm{H}$ NMR (400 MHz, $\mathrm{CDCl}_{3}$ ): $\delta=7.48-7.28$ (m, 5H), 6.96 (m, 1H), $3.96(\mathrm{~s}, 2 \mathrm{H})$.

${ }^{13} \mathrm{C}$ NMR $\left(101 \mathrm{MHz}, \mathrm{CDCl}_{3}\right): \delta=167.40,132.14,129.21,128.82,127.70,103.44,103.21$, $102.98,40.22,24.67$.

${ }^{19} \mathrm{~F}$ NMR $\left(376 \mathrm{MHz}, \mathrm{CDCl}_{3}\right): \delta=-138.76--139.35(\mathrm{~m}),-152.23--153.98(\mathrm{~m})$.

HRMS (EI): $m / z$ calcd for $[\mathrm{M}]^{+} \mathrm{C}_{14} \mathrm{H}_{8} \mathrm{~F}_{4} \mathrm{O}_{2}: 284.05$. Found: 284.05

Pentafluorophenyl 2-phenylacetate (4): Prepared according to the General Procedure. The title 1 compounds was obtained $(244 \mathrm{mg}, 0.81 \mathrm{mmol}, 49 \%)$ as a colorless oil following purification by column chromatography $\left[\mathrm{SiO}_{2}\right.$, Petroleum Ether: Ethyl Acetate 5:1].

Spectroscopic data was consistent with the literature report. ${ }^{5}$

Phenyl 2-phenylacetate (5): Prepared according to the General Procedure. The title compounds was obtained (301 $\mathrm{mg}, 1.42 \mathrm{mmol}, 86 \%$ ) as a white solid following 急至 purification by column chromatography $\left[\mathrm{SiO}_{2}\right.$, Petroleum Ether-EtOAc, 200:1].

Spectroscopic data was consistent with literature report. ${ }^{6}$ 


\section{Preparation of Allyl Partners - Table 1.}

Allyl chloride (CAS: 107-05-1) and allyl acetate (CAS: 591-87-7) were purchased from SigmaAldrich, USA and used as received.

Allyl Methanesulfonate (S1): A dry $100 \mathrm{~mL}$ round-bottom flask containing dry $\mathrm{CH}_{2} \mathrm{Cl}_{2}(25 \mathrm{~mL})$ was cooled to $0{ }^{\circ} \mathrm{C}$ using an ice bath. Allyl alcohol (4.0 mL, $58.7 \mathrm{mmol}, 1.0$ equiv.) $\sim^{0} \cdot s^{\prime \prime}=0$ and triethylamine $(9.0 \mathrm{~mL}, 64.5 \mathrm{mmol}, 1.1$ equiv.) were added sequentially via $\mathrm{Me} \quad$ syringe. Methanesulfonic anhydride (11.25 g, $64.5 \mathrm{mmol}, 1.1$ equiv.) was added in small portions over $15 \mathrm{~min}$ and the reaction was allowed to warm to room temperature and stir for $24 \mathrm{~h}$. The reaction was transferred directly to a separatory funnel and washed with water $(5 \times$ $25 \mathrm{~mL})$. The organic layer was dried $\left(\mathrm{MgSO}_{4}\right)$ and concentrated under reduced pressure giving a light brown liquid. The residue (light brown liquid) was purified by short-path vacuum distillation (3 mmg Hg, b.p. $=70-74{ }^{\circ} \mathrm{C}$ to provide the title compound (S1) (3.59 g, $26.3 \mathrm{mmol}$, $45 \%)$ as a clear colorless liquid.

Spectroscopic data was consistent with the literature report. ${ }^{7}$

Allyl diethyl phosphate (S2): Prepared according to the procedure of Hosomi and co-workers. ${ }^{8}$<smiles>C=CCOP(C)OCC</smiles>

Ö

Allyl diphenyl phosphate (S3): Prepared according to the procedure of O'Brien and co-workers. ${ }^{9}$<smiles>C=CCO[Pb](=O)O</smiles>

Allyl isobutyl carbonate (S4): Allyl alcohol (9.50 mL, $140 \mathrm{mmol}, 1.4$ equiv.) and $\mathrm{CH}_{2} \mathrm{Cl}_{2}$ (60 $\mathrm{mL})$ were added to a dry $100 \mathrm{~mL}$ round bottom flask under an atmosphere of
nitrogen. Dry pyridine $(24.2 \mathrm{~mL}, 300 \mathrm{mmol})$ was added and resulting solution
cooled $0{ }^{\circ} \mathrm{C}$ using an ice bath. iso-Butyl chloroformate $(13.0 \mathrm{~mL}, 100 \mathrm{mmol}$, 1.0 equiv.) was added dropwise via syringe and the reaction was allowed to warm slowly to r.t. and stirred for $12 \mathrm{~h}$. The reaction was filtered to remove the precipitate and the filtrate washed sequentially with sat. aq. $\mathrm{NH}_{4} \mathrm{Cl}, \mathrm{H}_{2} \mathrm{O}$ and sat. aq. brine solution. The organic layer was dried $\left(\mathrm{MgSO}_{4}\right)$ and concentrated under reduced pressure. The residue was purified column chromatography $\left(\mathrm{SiO}_{2}\right.$, Petroleum Ether-EtOAc, 9:1) to give the title compound (S4) as a colorless oil (12.06 g, $76.3 \mathrm{mmol}, 76 \%)$.

${ }^{1} \mathrm{H}$ NMR (400 MHz, $\left.\mathrm{CDCl}_{3}\right): \delta=5.89(\mathrm{ddt}, J=17.2,10.4,5.8 \mathrm{~Hz}, 1 \mathrm{H}), 5.31(\mathrm{dd}, J=17.2,1.4$ $\mathrm{Hz}, 1 \mathrm{H}), 5.21(\mathrm{dd}, J=10.4,1.4 \mathrm{~Hz}, 1 \mathrm{H}), 4.57$ (dt, $J=5.8,1.4 \mathrm{~Hz}, 2 \mathrm{H}), 3.88(\mathrm{~d}, J=6.7 \mathrm{~Hz}, 2 \mathrm{H})$, $1.93(\mathrm{dp}, J=13.4,6.7 \mathrm{~Hz}, 1 \mathrm{H}), 0.90(\mathrm{~s}, 6 \mathrm{H})$.

${ }^{13} \mathrm{C} \mathrm{NMR}\left(101 \mathrm{MHz}, \mathrm{CDCl}_{3}\right) \delta 155.18,131.79,118.72,74.10,68.28,27.82,18.90$. 
Allyl pivalate (S5): Allyl alcohol $(9.50 \mathrm{~mL}, 140 \mathrm{~mol})$ and $\mathrm{CH}_{2} \mathrm{Cl}_{2}(60 \mathrm{~mL})$ were added to a dry $100 \mathrm{~mL}$ round bottom flask under an atmosphere of nitrogen. Dry pyridine $(24.2$ $\mathrm{mL}, 300 \mathrm{mmol}, 3.0$ equiv.) and resulting solution cooled $0{ }^{\circ} \mathrm{C}$ using an ice bath. Pivaloyl chloride (12.3 mL, $100 \mathrm{mmol}, 1.0$ equiv.) was added dropwise via syringe and the reaction was allowed to warm slowly to r.t. and stirred for $12 \mathrm{~h}$. The reaction was filtered to remove the precipitate and the filtrate washed sequentially with sat. aq. $\mathrm{NH}_{4} \mathrm{Cl}, \mathrm{H}_{2} \mathrm{O}$ and sat. aq. brine solution. The organic layer was dried $\left(\mathrm{MgSO}_{4}\right)$ and concentrated under reduced pressure. The residue was purified column chromatography $\left(\mathrm{SiO}_{2}\right.$, Petroleum Ether-EtOAc, 9:1) to give the title compound (S5) as a colorless oil (11.51 g, 81.0 mmol, 81\%).

Spectroscopic data was consistent with the literature report. ${ }^{10}$

tert-Butyl allyl carbonate (S6): Prepared according to the procedure of Hartwig and co$\gamma^{0} \pi_{0}^{0} K$ workers. $^{11}$ 
Assessment of selected amine catalysts.

Using standard conditions with pentafluorophenyl ester and allyl mesylate.
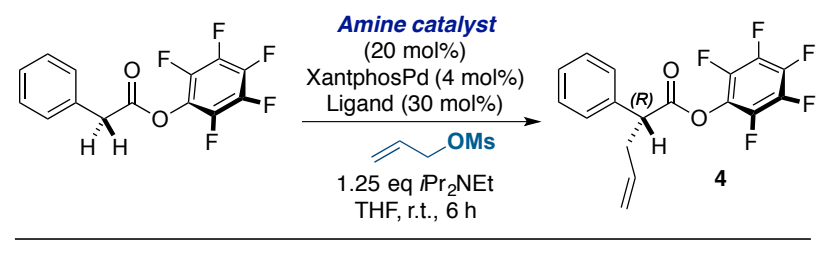

\begin{tabular}{cc} 
Entry & Amine Catalys \\
\hline 1 & none \\
2 & $\mathrm{NEt}_{3}$ \\
3 & $\mathrm{DMAP}^{c}$ \\
4 & OTMS-qunine
\end{tabular}

Yield $[\%]^{a} \quad e e[\%]^{b}$

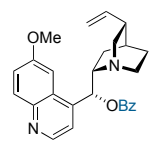

6

OBz-quinine

2 --

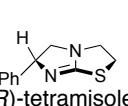

2

4

5

7

7

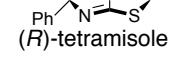

30

35

8

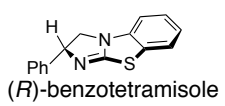
(BTM)

91

92

9

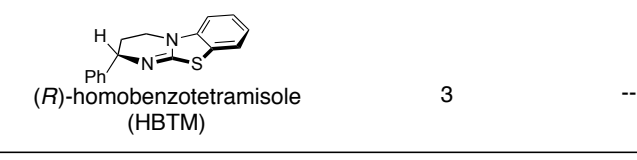

aYields determined by $1 \mathrm{H}-\mathrm{NMR}$ comparison with an internal standard (1,2,4,5-tetramethylbenzene).

${ }^{b}$ Determined by Chiral HPLC analysis in comparison with the racemate.

${ }^{c} 4$-(dimethylamino)pyridine.

d4-pyrrolidinopyridine.

Only the isothiourea catalysts (entries $7 \& 8$ ) were effective. Surprisingly, HBMT (entry 9) was not an efficient catalyst. 
Assessment of selected leaving groups $(\mathrm{X})$ for cinnamyl substrates

Using optimized conditions.

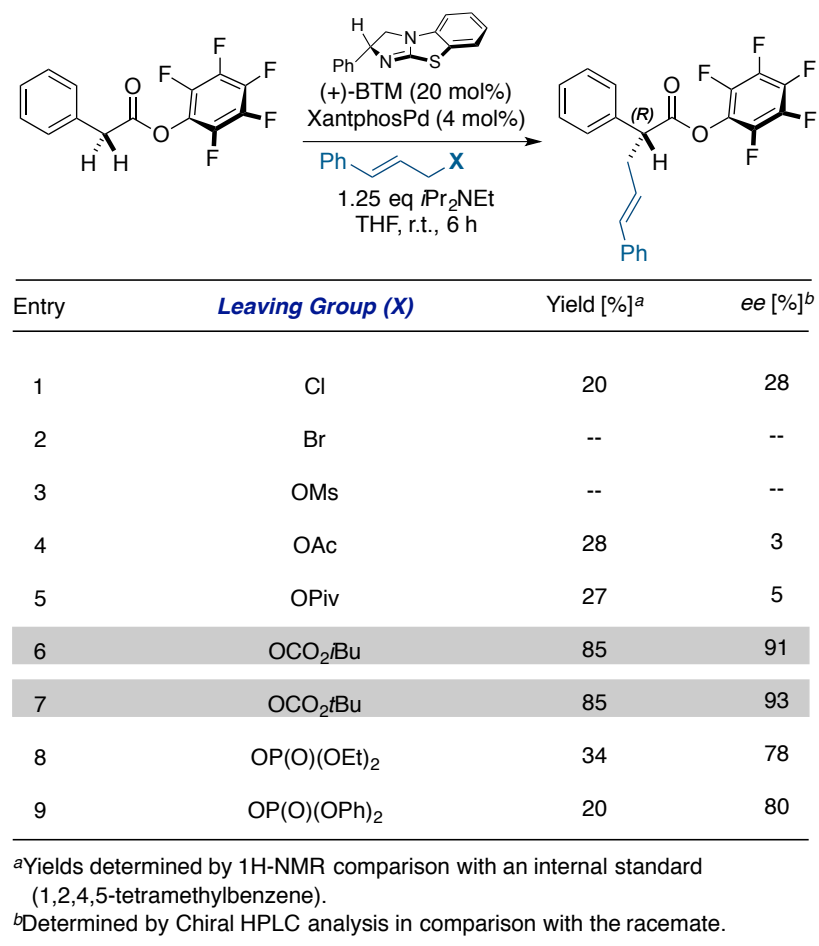




\section{Preparation of Esters - Table 2.}

General Procedure A: The specified phenylacetic acid (1.65 mmol, 1.0 equiv.), pentafluorophenol (0.36 g, $1.82 \mathrm{mmol}, 1.1$ equiv.) and dry $\mathrm{CH}_{2} \mathrm{Cl}_{2}$ (40 mL) were added sequentially to a dry round-bottom flask at r.t. under an atmosphere of nitrogen. The reaction was cooled to $0{ }^{\circ} \mathrm{C}$ using an ice bath and DCC (0.37 g, $1.82 \mathrm{mmol}, 1.1$ equiv.) and DMAP (20 mg, $0.165 \mathrm{mmol}, 0.1$ equiv.) added sequentially. The reaction was allowed to slowly warm to room temperature and further stirred for 16 hours. Thereafter, aq. $\mathrm{HCl}(3 N, 2.0 \mathrm{~mL})$ was added and the reaction placed in a freezer $\left(-20^{\circ} \mathrm{C}\right)$ for a minimum of $6 \mathrm{~h}$ The resulting suspension was filtered over celite and the residue washed with ice-cold $\mathrm{CH}_{2} \mathrm{Cl}_{2}$. The combined filtrates were successively washed with sat. aq. $\mathrm{NaHCO}_{3}$ and water before being dried $\left(\mathrm{MgSO}_{4}\right)$ and concentrated under reduced pressure. The residue was purified by column chromatography $\left[\mathrm{SiO}_{2}\right.$, specified eluent].

General Procedure B: The specified phenylacetic acid (1.65 mmol, 1.0 equiv.), pentafluorophenol (0.36 g, $1.82 \mathrm{mmol}, 1.1$ equiv.) and dry $\mathrm{CH}_{2} \mathrm{Cl}_{2}(20 \mathrm{~mL})$ were added sequentially to a dry round-bottom flask at r.t. under an atmosphere of nitrogen. Pyridine (0.15 $\mathrm{mL}, 1.82 \mathrm{mmol}, 1.1 \mathrm{eq})$ and pentafluorophenyl trifluoroacetate $(0.35 \mathrm{~mL}, 2.11 \mathrm{mmol}, 1.16 \mathrm{eq})$ were sequentially added. The reaction was stirred for $16 \mathrm{~h}$. Thereafter, the reaction mixture was transferred to a separatory funnel and was washed sequentially with aq. $\mathrm{HCl}(0.1 \mathrm{~N})$, sat. aq. $\mathrm{NaHCO}_{3}$ and $\mathrm{H}_{2} \mathrm{O}$. The organic layer was dried $\left(\mathrm{MgSO}_{4}\right)$ and concentrated under reduced pressure. The residue was purified by column chromatography $\left[\mathrm{SiO}_{2}\right.$, specified eluent $]$.

Pentafluorophenyl 2-(4-methylphenyl)acetate (S7): Prepared according to General Procedure

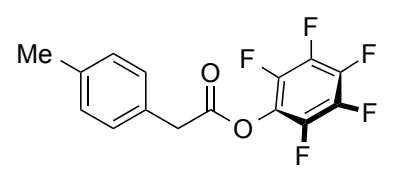

A. The title compounds was obtained $(255 \mathrm{mg}, 0.80 \mathrm{mmol}, 48 \%)$ as a colorless oil following purification by column chromatography $\left[\mathrm{SiO}_{2}\right.$, Petroleum Ether: Ethyl Acetate 5:1].

IR (film): 3028, 2928, 1790, 1655, $1520 \mathrm{~cm}^{-1}$.

${ }^{1} \mathrm{H}$ NMR (400 MHz, $\left.\mathrm{CDCl}_{3}\right): \delta=7.26(\mathrm{~d}, J=8,2 \mathrm{H}), 7.20(\mathrm{~d} J=8,2 \mathrm{H}), 3.92(2 \mathrm{H}, \mathrm{s}), 2.35(3 \mathrm{H}$, s).

${ }^{13} \mathrm{C}$ NMR (400 MHz, $\left.\mathrm{CDCl}_{3}\right): \delta=167.8,142.4,140.7,139.8,139.1,137.5,129.5,129.0,39.7$, 21.0.

${ }^{19} \mathrm{~F}$ NMR (376 MHz, $\left.\mathrm{CDCl}_{3}\right): \delta=-152.59--153.71(\mathrm{~m}),-158.19(\mathrm{t}, J=21.4 \mathrm{~Hz}),-162.23--$ $163.21(\mathrm{~m})$.

HRMS (CI): $m / z$ calcd for $[\mathrm{M}]^{+} \mathrm{C}_{15} \mathrm{H}_{9} \mathrm{~F}_{5} \mathrm{O}_{2}: 316.05$. Found 316.05

Pentafluorophenyl 2-(4-methoxyphenyl)acetate (S8): Prepared according to General Procedure A. The title compound was obtained (479 $\mathrm{mg}, 1.44 \mathrm{mmol}, 87 \%)$ as a<smiles>COc1ccc(CC(=O)Oc2c(F)c(F)c(F)c(F)c2F)cc1</smiles>
colorless oil following purification by column chromatography $\left[\mathrm{SiO}_{2}\right.$, Petroleum Ether: Ethyl Acetate 5:1]. 
IR (film): 2935, 1788, 1613, $1519 \mathrm{~cm}^{-1}$.

${ }^{1} \mathrm{H}$ NMR $\left(400 \mathrm{MHz}, \mathrm{CDCl}_{3}\right): \delta=7.27(\mathrm{~d}, J=8.9 \mathrm{~Hz}, 2 \mathrm{H}), 6.91(\mathrm{~d}, J=8.7 \mathrm{~Hz}, 1 \mathrm{H}), 3.91(\mathrm{~s}, 2 \mathrm{H})$, $3.82(\mathrm{~s}, 3 \mathrm{H})$.

${ }^{13} \mathrm{C} \mathrm{NMR}\left(101 \mathrm{MHz}, \mathrm{CDCl}_{3}\right): \delta=167.74,159.15,130.28,123.96,114.25,55.24,39.28$.

${ }^{19} \mathrm{~F}$ NMR (376 MHz, $\left.\mathrm{CDCl}_{3}\right): \delta=-152.38--152.95(\mathrm{~m}),-158.01(\mathrm{t}, J=21.6 \mathrm{~Hz}),-162.10--$ $162.92(\mathrm{~m})$.

HRMS (CI): $m / z$ calcd for $\left[\mathrm{M}^{+}\right] \mathrm{C}_{15} \mathrm{H}_{9} \mathrm{~F}_{5} \mathrm{O}_{3}: 332.05$. Found: 332.05 .

Pentafluorophenyl 2-(4- chlorophenyl)acetate (S9): Prepared according to General Procedure

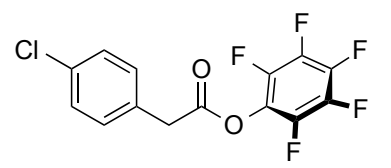

A. The title compounds was obtained (398 $\mathrm{mg}, 1.18 \mathrm{mmol}, 72 \%)$ as a colorless oil following purification by column chromatography $\left[\mathrm{SiO}_{2}\right.$, Petroleum Ether: Ethyl Acetate 5:1].

IR (film): 2925, 1789, 1519, $1494 \mathrm{~cm}^{-1}$.

${ }^{1} \mathrm{H}$ NMR (600 MHz, $\left.\mathrm{CDCl}_{3}\right): \delta=7.36(\mathrm{~d}, J=8.4 \mathrm{~Hz}, 1 \mathrm{H}), 7.30(\mathrm{~d}, J=8.4 \mathrm{~Hz}, 2 \mathrm{H}), 3.95(\mathrm{~s}, 2 \mathrm{H})$.

${ }^{13} \mathrm{C} \mathrm{NMR}\left(100 \mathrm{MHz}, \mathrm{CDCl}_{3}\right): \delta=167.1,133.9,130.6,130.4,129.0,39.4$.

${ }^{19} \mathrm{~F}$ NMR (376 MHz, $\left.\mathrm{CDCl}_{3}\right): \delta=-151.81--153.18(\mathrm{~m}),-157.66(\mathrm{t}, J=21.6 \mathrm{~Hz}),-161.08--$ $164.20(\mathrm{~m})$.

HRMS (CI): $m / z$ calcd for $[\mathrm{M}+\mathrm{H}]^{+} \mathrm{C}_{14} \mathrm{H}_{7} \mathrm{~F}_{5} \mathrm{O}_{2} \mathrm{Cl}: 337.00$. Found: 337.00

Pentafluorophenyl 2-(o-tolyl)acetate (S10): Prepared according to General Procedure A. The

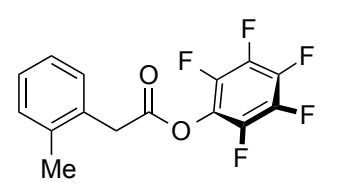
title compounds was obtained (177 $\mathrm{mg}, 0.56 \mathrm{mmol}, 34 \%)$ as a colorless oil following purification by column chromatography $\left[\mathrm{SiO}_{2}\right.$, Petroleum Ether: Ethyl Acetate 5:1].

IR (film): 1761, 1592, 1492, 1456, 1339, 1217, 1196, 1127, 747, $696 \mathrm{~cm}^{-1}$.

${ }^{1} \mathrm{H}$ NMR (400 MHz, $\left.\mathrm{CDCl}_{3}\right): \delta=7.31-7.16(\mathrm{~m}, 4 \mathrm{H}), 3.96$ (s, 2H), 2.36 (s, 3H).

${ }^{13} \mathrm{C}$ NMR (101 MHz, $\left.\mathrm{CDCl}_{3}\right): \delta=167.36,136.89,130.76,130.57,130.21,128.10,126.39$, $63.73,38.10,19.34$.

${ }^{19} \mathrm{~F}$ NMR $\left(376 \mathrm{MHz}, \mathrm{CDCl}_{3}\right): \delta=-152.51--152.98(\mathrm{~m}),-157.97(\mathrm{t}, J=21.7 \mathrm{~Hz}),-162.10--$ $162.78(\mathrm{~m})$.

HRMS (CI): $m / z$ calcd for $[\mathrm{M}]^{+} \mathrm{C}_{15} \mathrm{H}_{9} \mathrm{~F}_{5} \mathrm{O}_{2}: 316.05$. Found 316.05 . 
Pentafluorophenyl 2-(2-bromo-3,4-dimethoxyphenyl)acetate (S11): Prepared according to

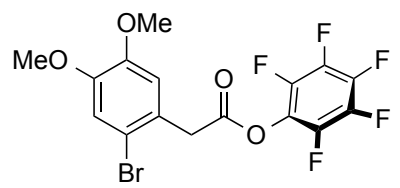
General Procedure A. The title compounds was obtained $(500 \mathrm{mg}$, $1.13 \mathrm{mmol}, 68 \%$ ) as a white solid following purification by column chromatography $\left[\mathrm{SiO}_{2}\right.$, Petroleum Ether: Ethyl Acetate 5:1].

M.p. (pentane-EtOAc, 400:1; needles) $=97-99^{\circ} \mathrm{C}$

IR (film): 1787.1, 1520.2, 1093.0, $1000.25 \mathrm{~cm}^{-1}$

${ }^{1} \mathrm{H}$ NMR (400 MHz, $\left.\mathrm{CDCl}_{3}\right): \delta=7.05$ (s, 1H), 6.85 (s, 1H), 4.05 (s, 2H), $3.86(\mathrm{~s}, 6 \mathrm{H})$.

${ }^{13} \mathrm{C}$ NMR $\left(100 \mathrm{MHz}, \mathrm{CDCl}_{3}\right): \delta=166.76,149.27,148.55,142.40,142.36,142.27,140.73$, $139.87,139.73,139.06,138.21,136.55,124.99$, 123.99, 115.49, 115.00, 113.58, 56.09, 56.01, 40.12 .

${ }^{19} \mathrm{~F}$ NMR $\left(376 \mathrm{MHz}, \mathrm{CDCl}_{3}\right): \delta=-152.00--152.92(\mathrm{~m}),-157.69(\mathrm{t}, J=21.7 \mathrm{~Hz}),-162.14(\mathrm{td}, J$ $=22.4,5.0 \mathrm{~Hz})$.

HRMS (EI): $m / z$ calcd for $[\mathrm{M}+\mathrm{H}]^{+} \mathrm{C}_{16} \mathrm{H}_{11} \mathrm{BrF}_{5} \mathrm{O}_{2}$ : 440.98. Found: 440.98 .

Pentafluorophenyl 2-(4-vinylphenyl)acetate (S12): Prepared according to General Procedure B. The title compounds was obtained (44 mg, 0.14 mmol, 40\%) as an orange
oil following purification by column chromatography $\left[\mathrm{SiO}_{2}\right.$, Petroleum
Ether: Ethyl Acetate 5:1].

IR (film): 557, 738, 996, 1020, 1143, 1516, 1788, $2963 \mathrm{~cm}^{-1}$

${ }^{1} \mathrm{H}$ NMR (400 MHz, $\left.\mathrm{CDCl}_{3}\right): \delta=7.51-7.40(\mathrm{~m}, 2 \mathrm{H}), 7.37-7.28(\mathrm{~m}, 2 \mathrm{H}), 6.72(\mathrm{dd}, J=17.7,11.0$ $\mathrm{Hz}, 1 \mathrm{H}), 5.77(\mathrm{dd}, J=17.7,0.9 \mathrm{~Hz}, 1 \mathrm{H}), 5.28(\mathrm{dd}, J=11.0,0.9 \mathrm{~Hz}, 1 \mathrm{H}), 3.96(\mathrm{~s}, 2 \mathrm{H})$.

${ }^{13} \mathrm{C} \mathrm{NMR}\left(400 \mathrm{MHz}, \mathrm{CDCl}_{3}\right): \delta=169.71,136.16,126.65,114.31,39.85$.

${ }^{19} \mathrm{~F}$ NMR (376 MHz, $\left.\mathrm{CDCl}_{3}\right): \delta=-152.63(\mathrm{~d}, J=17.4 \mathrm{~Hz}),-157.87(\mathrm{t}, J=21.7 \mathrm{~Hz}),-162.10--$ $162.53(\mathrm{~m})$.

HRMS (EI): $m / z$ calc for $[\mathrm{M}]^{+} \mathrm{C}_{16} \mathrm{H}_{9} \mathrm{~F}_{5} \mathrm{O}_{2}: 328.05$. Found: 328.05 .

Pentafluorophenyl 2-(2-methoxyphenyl)acetate (S13): Prepared according to General

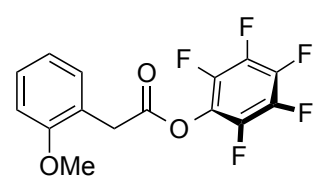
Procedure A. The title compounds was obtained (225 mg, $0.68 \mathrm{mmol}, 41 \%$ ) as a colorless oil following purification by column chromatography $\left[\mathrm{SiO}_{2}\right.$, Petroleum Ether: Ethyl Acetate 5:1].

IR (film): 2935, 1788, 1613, $1519 \mathrm{~cm}^{-1}$.

${ }^{1} \mathrm{H}$ NMR (400 MHz, $\left.\mathrm{CDCl}_{3}\right): \delta=7.34(\mathrm{t}, J=7.9,1 \mathrm{H}), 7.27(\mathrm{~m}, 1 \mathrm{H}), 7.04-6.87(\mathrm{~m}, 2 \mathrm{H}), 3.95(\mathrm{~s}$, $2 \mathrm{H}), 3.87(\mathrm{~s}, 3 \mathrm{H})$.

${ }^{13} \mathrm{C}$ NMR (101 MHz, $\left.\mathrm{CDCl}_{3}\right): \delta=167.75,157.49,130.81,129.30,121.32,120.56,110.33$, 57.76, 56.02, 55.27, 54.77, 35.47. 
${ }^{19} \mathrm{~F}$ NMR (376 MHz, $\left.\mathrm{CDCl}_{3}\right): \delta=-152.47--153.26(\mathrm{~m}),-158.39(\mathrm{t}, J=21.7 \mathrm{~Hz}),-162.69(\mathrm{td}, J$ $=22.1,4.7 \mathrm{~Hz}$ ).

HRMS (CI): $m / z$ calcd for $[\mathrm{M}]^{+} \mathrm{C}_{15} \mathrm{H}_{9} \mathrm{~F}_{5} \mathrm{O}_{3}: 332.05$. Found: 332.05 .

Pentafluorophenyl 2-(naphthalene-2-yl)acetate (S14): Prepared according to General Procedure A. The title compounds was obtained (401 mg, 1.19 mmol,
$72 \%)$ as a white solid following purification by column chromatography
$\left[\mathrm{SiO}_{2}\right.$, Petroleum Ether: Ethyl Acetate 5:1].

M.p. (pentane-EtOAc, 400:1; needles) $=81-82{ }^{\circ} \mathrm{C}$

IR (film): 3052.93, 2926.10, 1789.49, $1519.34 \mathrm{~cm}^{-1}$

${ }^{1} \mathrm{H}$ NMR (400 MHz, $\left.\mathrm{CDCl}_{3}\right): \delta=7.88-7.83(4 \mathrm{H}, \mathrm{m}), 7.53-7.46(3 \mathrm{H}, \mathrm{m}), 4.34(2 \mathrm{H}, \mathrm{s})$.

${ }^{13} \mathrm{C} \mathrm{NMR}\left(100 \mathrm{MHz}, \mathrm{CDCl}_{3}\right): \delta=167.4,133.4,132.7,129.4,128.7,128.3,127.7,126.8,126.4$, 126.2, 40.3.

${ }^{19} \mathrm{~F}$ NMR $\left(376 \mathrm{MHz}, \mathrm{CDCl}_{3}\right): \delta=-151.31--155.39(\mathrm{~m}),-157.82(\mathrm{t}, J=21.7 \mathrm{~Hz}),-161.80--$ 162.94 (m).

HRMS (EI): $m / z$ calcd for $[\mathrm{M}]^{+} \mathrm{C}_{18} \mathrm{H}_{9} \mathrm{~F}_{5} \mathrm{O}_{2}: 352.05$. Found: 352.05 .

Pentafluorophenyl 2-(2,3,4-trifluorophenyl)acetate (S15): Prepared according to General F Procedure A. The title compounds was obtained (176 mg, $0.49 \mathrm{mmol}$, 30\%) as a colorless oil following purification by column chromatography [SiO ${ }_{2}$, Petroleum Ether: Ethyl Acetate 5:1].

IR (film): 1791.0, 1519.92, 1492.6, $1104.3,1001.9 \mathrm{~cm}^{-1}$

1H NMR (400 MHz, $\mathrm{CDCl}_{3}$ ): $\delta=7.13-6.94(\mathrm{~m}, 2 \mathrm{H}), 4.01$ (s, 2H).

${ }^{13} \mathrm{C} \mathrm{NMR}\left(100 \mathrm{MHz}, \mathrm{CDCl}_{3}\right): \delta=165.95,124.61(\mathrm{dt}, J=8.3,4.1 \mathrm{~Hz}), 112.41(\mathrm{dd}, J=17.6,4.0$ $\mathrm{Hz}), 77.21,32.97,29.72,1.03$.

${ }^{19}$ F NMR (376 MHz, $\left.\mathrm{CDCl}_{3}\right): \delta=-133.42--134.34(\mathrm{~m}),-135.98--137.16(\mathrm{~m}),-152.14--$ $153.27(\mathrm{~m}),-157.28$ (t, $J=21.7 \mathrm{~Hz}),-159.39$ (tdd, $J=20.1,7.0,2.3 \mathrm{~Hz}),-161.75--162.38(\mathrm{~m})$.

HRMS (CI): $m / z$ calcd for $[\mathrm{M}+\mathrm{H}]^{+} \mathrm{C}_{14} \mathrm{H}_{5} \mathrm{~F}_{8} \mathrm{O}_{2}: 357.02$. Found: 357.02 .

Pentafluorophenyl 2-(4-isopropylphenyl)acetate (S16): Prepared according to General F Procedure A. The title compounds was obtained (214 mg, $0.62 \mathrm{mmol}$, $38 \%)$ as a yellow oil following purification by column chromatography
$\left[\mathrm{SiO}_{2}\right.$, Petroleum Ether: Ethyl Acetate 10:1]. IR (film): 738, 997, 1085, 1517, 1704, 1787, $2963 \mathrm{~cm}^{1}$. 
${ }^{1} \mathrm{H}$ NMR (400 MHz, $\left.\mathrm{CDCl}_{3}\right): \delta=7.29(\mathrm{q}, J=8.4 \mathrm{~Hz}, 2 \mathrm{H}), 7.25(\mathrm{q}, J=8.4 \mathrm{~Hz}, 2 \mathrm{H}) 3.95(\mathrm{~s}, 2 \mathrm{H})$, 2.93 (hept, $J=6.9 \mathrm{~Hz}, 1 \mathrm{H}), 1.27$ (d, $J=6.9 \mathrm{~Hz}, 7 \mathrm{H})$.

${ }^{13} \mathrm{C} \mathrm{NMR}\left(100 \mathrm{MHz}, \mathrm{CDCl}_{3}\right): \delta=167.66,148.48,129.28,129.13,126.96,39.74,33.81,23.92$.

${ }^{19} \mathrm{~F}$ NMR $\left(376 \mathrm{MHz}, \mathrm{CDCl}_{3}\right): \delta=-152.38--152.79(\mathrm{~m}),-158.03(\mathrm{t}, J=21.7 \mathrm{~Hz}),-162.27--$ $162.85(\mathrm{~m})$.

HRMS (CI): $m / z$ calcd for $[\mathrm{M}+\mathrm{H}]^{+} \mathrm{C}_{17} \mathrm{H}_{14} \mathrm{~F}_{5} \mathrm{O}_{2}: 345.09$. Found: 345.09 .

Indomethacin-pentafluorophenyl ester (S17): Prepared according to General Procedure A. The Cl- title compounds was obtained (467 mg, $0.89 \mathrm{mmol}, 54 \%$ ) as a 2. [SiO 2 , Petroleum Ether: Ethyl Acetate 10:1].

$\mathrm{MeO}$

M.p. (pentane-EtOAc, 400:1; needles) $=130-131^{\circ} \mathrm{C}$

IR (film): 3439, 2931, 1786, 1686, 1592, $1521 \mathrm{~cm}^{-1}$.

${ }^{1} \mathrm{H}$ NMR $\left(500 \mathrm{MHz}, \mathrm{CDCl}_{3}\right): \delta=7.67(\mathrm{~d}, J=8.5 \mathrm{~Hz}, 2 \mathrm{H}), 7.48(\mathrm{~d}, J=8.5 \mathrm{~Hz}, 2 \mathrm{H}), 6.97(\mathrm{~d}, J=$ $2.5 \mathrm{~Hz}, 1 \mathrm{H}), 6.89$ (d, $J=9.0 \mathrm{~Hz}, 1 \mathrm{H}), 6.71(\mathrm{dd}, J=9.0,2.5 \mathrm{~Hz}, 1 \mathrm{H}), 4.02(\mathrm{~s}, 2 \mathrm{H}), 3.85(\mathrm{~s}, 3 \mathrm{H})$, $2.44(\mathrm{~s}, 3 \mathrm{H})$.

${ }^{13} \mathrm{C} \mathrm{NMR}\left(126 \mathrm{MHz}, \mathrm{CDCl}_{3}\right): \delta=166.8,156.2,139.5,136.5,133.7,131.2,130.8,130.1,129.2$, $115.1,112.1,110.6,100.8,55.7,29.3,13.3$.

${ }^{19} \mathrm{~F}$ NMR $\left(376 \mathrm{MHz}, \mathrm{CDCl}_{3}\right): \delta=-152.32--153.04(\mathrm{~m}),-157.63(\mathrm{t}, J=21.6 \mathrm{~Hz}),-160.93--$ $162.77(\mathrm{~m})$.

HRMS (CI): $m / z$ calcd for $[\mathrm{M}+\mathrm{H}]^{+} \mathrm{C}_{25} \mathrm{H}_{15} \mathrm{ClF}_{5} \mathrm{NO}_{4}$ : 524.07, Found: 524.07.

Preparation of Cinnamyl tert-butylcarbonates - Table 2.

The following tert-butylcarbonates were prepared following literature procedures and spectroscopic data was in accordance with those reports.

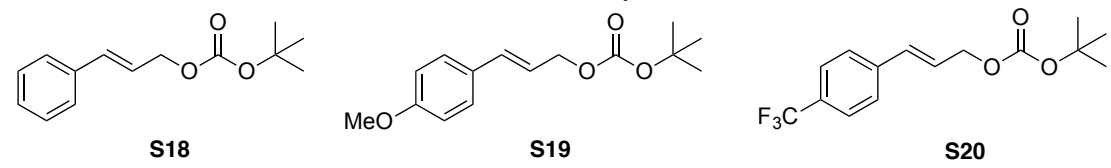

$\mathrm{S}_{18}{ }^{12}$

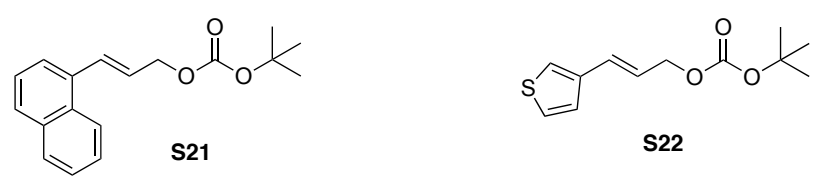

$\mathrm{S} 19$ \& $\mathrm{S20}^{11}$

$\mathbf{S} 21 \& \mathbf{S 2 2}^{13}$ 
(E)-tert-Butyl 3-(3-chloro-4-methoxyphenyl)allyl carbonate (S23): Using a modification of the o procedures of Hartwig and co-workers and Fréchet and co-workers for preparing similar compounds. ${ }^{11,14,15} \mathrm{~A} 50 \mathrm{~mL}$ round bottom flask was charged with di-tert-butyl carbonate $(3.05 \mathrm{~g}, 14.0 \mathrm{mmol}, 1.4$ equiv.) and $\mathrm{CH}_{2} \mathrm{Cl}_{2}(2.6 \mathrm{~mL})$. To the resulting colorless solution was added (E)-tert-butyl 3-(3-chloro-4-methoxyphenyl)allyl alcohol (3.05 g, 10.0 mmol, 1.0 equiv.) followed by $\mathrm{Bu}_{4} \mathrm{NHSO}_{4}(102 \mathrm{mg}, 0.3 \mathrm{mmol}, 0.03$ equiv.). The solution was then cooled to $0{ }^{\circ} \mathrm{C}$ using a ice bath, ice cold aq. $\mathrm{NaOH}(30 \% \mathrm{w} / \mathrm{w}, 5 \mathrm{~mL})$ added slowly with vigorous stirring and the stirring maintained for an additional $3 \mathrm{~h}$ whereupon TC indicated complete consumption of alcohol. Thereafter, the reaction was diluted with $\mathrm{CH}_{2} \mathrm{Cl}_{2}(30 \mathrm{~mL})$, the layers separated and the organic layer washed with sat. aq. Brine $(3 \times)$, dried $\left(\mathrm{MgSO}_{4}\right)$ and concentrated under reduced pressure. To remove remaining di-tert-butyl carbonate (using the procedure of Basel and Hassner), the resulting oil was dissolved in $\mathrm{EtOH}$, imidazole (340 $\mathrm{mg}$, $5.0 \mathrm{mmol}, 0.5$ equiv.) was added and the resulting solution stirred for $5 \mathrm{~min}$ before being concentrated under reduced pressure. The residue was purified by column chromatography [ $\mathrm{SiO}_{2}$, hexanes-EtOAc, 99:1] to afford the title compound $\mathbf{S 2 3}$ as a viscous colorless oil (2.53 g, $8.5 \mathrm{mmol}, 85 \%)$.

IR (film): 1740, 1585, 1129, 926, $730 \mathrm{~cm}^{-1}$.

${ }^{1} \mathrm{H}$ NMR $\left(500 \mathrm{MHz}, \mathrm{CDCl}_{3}\right): \delta=7.42(\mathrm{~d}, J=2.1 \mathrm{~Hz}, 1 \mathrm{H}), 7.23(\mathrm{dd}, J=8.5,2.1 \mathrm{~Hz}, 1 \mathrm{H}), 6.87(\mathrm{~d}$, $J=8.5 \mathrm{~Hz}, 1 \mathrm{H}), 6.55(\mathrm{dd}, J=15.8,1.4 \mathrm{~Hz}, 1 \mathrm{H}), 6.17(\mathrm{dt}, J=15.8,6.4 \mathrm{~Hz}, 1 \mathrm{H}), 4.69$ (dd, $J=6.5$, $1.4 \mathrm{~Hz}, 2 \mathrm{H}), 3.89(\mathrm{~s}, 3 \mathrm{H}), 1.50(\mathrm{~s}, 9 \mathrm{H})$.

${ }^{13} \mathrm{C} \mathrm{NMR}\left(126 \mathrm{MHz}, \mathrm{CDCl}_{3}\right) \delta 154.93,153.46,132.89,130.05,128.34,126.37,122.84,122.26$, $112.09,82.39,67.46,56.32,27.93$.

HRMS (CI): $m / z$ calcd for $[\mathrm{M}+\mathrm{H}]^{+} \mathrm{C}_{15} \mathrm{H}_{20} \mathrm{O}_{4} \mathrm{Cl}: 299.11$. Found: 299.11.

(E)-tert-Butyl 3-(4-((tert-butyldimethylsilyl)oxy)-3,5-dimethoxyphenyl)allyl carbonate (S24):

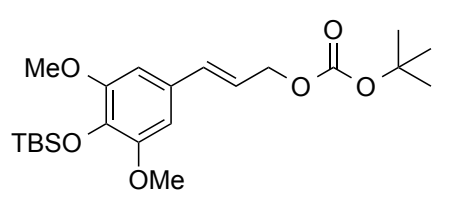

Using a modification of the procedures of Hartwig and co-workers and Fréchet and co-workers for preparing similar compounds. ${ }^{11,14,15}$ A $50 \mathrm{~mL}$ round bottom flask was charged with di-tert-butyl carbonate (3.05 g, 14.0 mmol, 1.4 equiv.) and $\mathrm{CH}_{2} \mathrm{Cl}_{2}(2.6 \mathrm{~mL})$. To the resulting colorless solution was added (E)-tert-butyl 3-(4-((tert-

butyldimethylsilyl)oxy)-3,5-dimethoxyphenyl)allyl alcohol (3.24 g, $10.0 \mathrm{mmol}, 1.0$ equiv.) followed by $\mathrm{Bu}_{4} \mathrm{NHSO}_{4}\left(102 \mathrm{mg}, 0.3 \mathrm{mmol}, 0.03\right.$ equiv.). The solution was then cooled to $0{ }^{\circ} \mathrm{C}$ using a ice bath, ice cold aq. $\mathrm{NaOH}(30 \% \mathrm{w} / \mathrm{w}, 5 \mathrm{~mL})$ added slowly with vigorous stirring and the stirring maintained for an additional $3 \mathrm{~h}$ whereupon TC indicated complete consumption of alcohol. Thereafter, the reaction was diluted with $\mathrm{CH}_{2} \mathrm{Cl}_{2}(30 \mathrm{~mL})$, the layers separated and the organic layer washed with sat. aq. Brine $(3 \times)$, dried $\left(\mathrm{MgSO}_{4}\right)$ and concentrated under reduced pressure. To remove remaining di-tert-butyl carbonate (using the procedure of Basel and Hassner), the resulting oil was dissolved in EtOH, imidazole (340 mg, $5.0 \mathrm{mmol}, 0.5 \mathrm{equiv}$.) was added and the resulting solution stirred for $5 \mathrm{~min}$ before being concentrated under reduced pressure. The residue was purified by column chromatography [ $\mathrm{SiO}_{2}$, hexanes-EtOAc, 99:1] to afford the title compound $\mathbf{S 2 4}$ as a viscous colorless oil (2.53 g, $8.5 \mathrm{mmol}, 85 \%)$. 
IR (film): 2929, 2855, 1735, 1581, 1518, 1464, 134, 1247, $1129 \mathrm{~cm}^{-1}$.

${ }^{1} \mathrm{H}$ NMR $\left(600 \mathrm{MHz}, \mathrm{CDCl}_{3}\right): \delta=7.29(\mathrm{~s}, 2 \mathrm{H}), 7.27(\mathrm{~d}, J=15.8 \mathrm{~Hz}, 1 \mathrm{H}), 6.86(\mathrm{dt}, J=15 . .8,6.6$ $\mathrm{Hz}, 1 \mathrm{H}), 5.40$ (dd $J=6.6,1.3 \mathrm{~Hz}, 1 \mathrm{H}), 4.49$ (s, 6H), 2.20 (s 9H), 0.82 (s, 6H).

${ }^{13} \mathrm{C}$ NMR $\left(151 \mathrm{MHz}, \mathrm{CDCl}_{3}\right): \delta=153.53,151.75,135.12,129.04,121.16,104.04,82.25,77.37$, $77.16,76.95,67.72,55.84,27.93,25.93,25.90,18.85,-4.52$.

HRMS (CI): $m / z$ calcd for $[\mathrm{M}+\mathrm{H}]^{+} \mathrm{C}_{22} \mathrm{H}_{37} \mathrm{O}_{6} \mathrm{Si}: 425.24$. Found: 425.24 .

\section{Preparation of product esters 6-24, Table 2.}

General Procedure C: XantphosPd (2.0 mg, $4.5 \mu \mathrm{mol}, 4 \mathrm{~mol} \%)$ was added to an oven-dried $4 \mathrm{~mL}$ vial containing a magnetic stir bar equipped with Teflon septa insert screw cap. The vial was evacuated and backfilled with nitrogen $(3 \times)$. Anhydrous THF $(0.5 \mathrm{~mL})$ was added via syringe and the resulting solution stirred at r.t. for $20 \mathrm{~min}$. Thereafter, the specified pentafluorophenyl ester (1.0 M in THF, $100 \mu \mathrm{L}, 0.1 \mathrm{mmol}, 1.0$ equiv.), $i \operatorname{Pr}_{2} \mathrm{NEt}$ (17 $\mu \mathrm{L}, 0.1 \mathrm{mmol}, 1.1$ equiv), (+)benzotetramisole $(0.4 \mathrm{M}$ in THF, $50 \mu \mathrm{L}, 20 \mu \mathrm{mol}, 20 \mathrm{~mol} \%)$ and the specified allyl mesylate or carbonate (1.0 M in THF, $125 \mu \mathrm{L}, 0.125 \mathrm{mmol}, 1.25$ equiv) were added sequentially via syringe and the reaction stirred at r.t. for $6 \mathrm{~h}$. The reaction was then diluted with pentane (complete vial volume) and filtered through activated, acidic $\mathrm{Al}_{2} \mathrm{O}_{3}$ (the vial and $\mathrm{Al}_{2} \mathrm{O}_{3}$ were washed $3 \times \mathrm{Et}_{2} \mathrm{O}$ ). The combined filtrates were concentrated and the residue purified by column chromatography $\left[\mathrm{SiO}_{2}\right.$, specified eluent].

Standard solutions (anhydrous THF) of the specified pentafluorophenyl ester, (+)benzotetramisole, and the specified allyl mesylate or carbonate were freshly prepared using volumetric flasks.

Reactions catalyzed by (+)-benzotetramisole yield the corresponding ( $R$ )-configured product esters. The absolute stereochemistry of the product esters was confirmed by the transformation of 6 to the corresponding alcohol, methyl ester and carboxylic acid, followed by comparison of their optical rotation (See page S26 for details). All other compounds have been assigned the (R)-configuration by analogy.

Pentaflourophenyl 2-phenylpent-4-enoate (6): Prepared according to General Procedure C. The

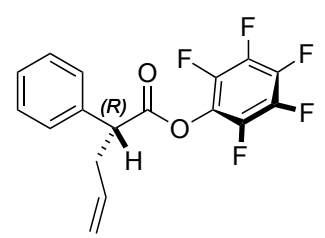
title compound 6 was obtained ( $30 \mathrm{mg}, 0.085 \mathrm{mmol}, 85 \%$ ) as a colorless oil following purification by column chromatography $\left[\mathrm{SiO}_{2}\right.$, Petroleum EtherEtOAc, 400:1]. The enantiomeric excess (92\% ee) was determined by chiral HPLC in comparison with the racemate (see below).

$$
[\alpha]_{\mathrm{D}}^{23}-66\left(c=0.17, \mathrm{CHCl}_{3}\right) .
$$

IR (film): 1784, 1520, 1092, $1008 \mathrm{~cm}^{-1}$.

${ }^{1} \mathrm{H}$ NMR (400 MHz, $\left.\mathrm{CDCl}_{3}\right): \delta=7.45-7.22(\mathrm{~m}, 5 \mathrm{H}), 5.79(\mathrm{~m}, 1 \mathrm{H}), 5.22-5.05(\mathrm{~m}, 2 \mathrm{H}), 4.00(\mathrm{dd}$, $J=8.6,6.7 \mathrm{~Hz}, 1 \mathrm{H}), 3.01-2.87(\mathrm{~m}, 1 \mathrm{H}), 2.70-2.58(\mathrm{~m}, 1 \mathrm{H})$.

${ }^{13} \mathrm{C} \mathrm{NMR}\left(101 \mathrm{MHz}, \mathrm{CDCl}_{3}\right): \delta=166.4,165.6,136.6,128.6,128.2,128.1,112.2,65.6,43.0$, $41.8,28.2,25.5,-1.05$. 
${ }^{19} \mathrm{~F} \mathrm{NMR}\left(376 \mathrm{MHz}, \mathrm{CDCl}_{3}\right): \delta=-151.90--152.98(\mathrm{~m}),-158.05(\mathrm{t}, J=21.8 \mathrm{~Hz}),-161.83--$ $163.80(\mathrm{~m})$.

HRMS (CI): $m / z$ calcd for $[\mathrm{M}]^{+} \mathrm{C}_{17} \mathrm{H}_{11} \mathrm{~F}_{5} \mathrm{O}_{2}: 342.17$. Found: 342.17 .

HPLC analysis using a chiral column (Chiralpak IA $3 \mu$ column, $22{ }^{\circ} \mathrm{C}, 0.5 \mathrm{~mL} / \mathrm{min}, 200: 1$ Hexane:Isopropanol, $210 \mathrm{~nm}, \mathrm{t}_{\text {minor }}=17.187 \mathrm{~min}, \mathrm{t}_{\text {major }}=21.424 \mathrm{~min}$ ).

Pentafluorophenyl 2-(4-methylphenyl)pent-4-enoate (7): Prepared according to General (R) Procedure C. The title compound 7 was obtained $(31 \mathrm{mg}, 0.086 \mathrm{mmol}$, $86 \%$ as a colorless oil following purification by column chromatography $\left[\mathrm{SiO}_{2}\right.$, Petroleum Ether-EtOAc, 400:1]. The enantiomeric excess $(92 \%$ ee) was determined by chiral HPLC in comparison with the racemate (see below).

$[\alpha]_{\mathrm{D}}^{23}-58.3\left(\mathrm{c}=0.76, \mathrm{CHCl}_{3}\right)$.

IR (film): 1777, 1519, $1091 \mathrm{~cm}^{-1}$.

${ }^{1} \mathrm{H}$ NMR (400 MHz, $\left.\mathrm{CDCl}_{3}\right): \delta=7.28(\mathrm{~d}, J=7.9 \mathrm{~Hz}, 2 \mathrm{H}), 7.20(\mathrm{~d}, J=7.9 \mathrm{~Hz}, 2 \mathrm{H}), 5.83-5.77$ (m, 1H), 5.26-4.95 (m, 2H), $3.98(\mathrm{dd}, J=8.6,6.8 \mathrm{~Hz}, 1 \mathrm{H}), 2.94(\mathrm{~m}, 1 \mathrm{H}), 2.64(\mathrm{~m}, 1 \mathrm{H}), 2.37$ (s, $3 \mathrm{H})$.

${ }^{13} \mathrm{C}$ NMR $\left(101 \mathrm{MHz}, \mathrm{CDCl}_{3}\right): \delta=169.8,137.9,134.4,133.9,129.8,128.0,118.0,77.5,77.2$, $76.8,50.7,37.5,21.3$.

${ }^{19} \mathrm{~F}$ NMR (376 MHz, $\left.\mathrm{CDCl}_{3}\right): \delta=-151.37--153.77(\mathrm{~m}),-158.13(\mathrm{t}, J=21.7 \mathrm{~Hz}),-162.53(\mathrm{td}, J$ $=22.1,4.9 \mathrm{~Hz})$.

HRMS (CI): $m / z$ calcd for $[\mathrm{M}+\mathrm{H}]^{+} \mathrm{C}_{18} \mathrm{H}_{14} \mathrm{~F}_{5} \mathrm{O}_{2}: 357.09$. Found: 357.09 .

HPLC analysis using a chiral column (Chiralpak IA $3 \mu$ column, $22{ }^{\circ} \mathrm{C}, 0.5 \mathrm{~mL} / \mathrm{min}, 200: 1$ Hexane:Isopropanol, $\left.210 \mathrm{~nm}, \mathrm{t}_{\text {minor }}=6.9 \mathrm{~min}, \mathrm{t}_{\text {major }}=7.3 \mathrm{~min}\right)$.

Pentafluorophenyl 2-(4-methoxyphenyl)pent-4-enoate (8): Prepared according to General II) Procedure C. The title compound $\mathbf{8}$ was obtained $(33 \mathrm{mg}, 0.088 \mathrm{mmol}$, $88 \%$ as a colorless oil following purification by column chromatography $\left[\mathrm{SiO}_{2}\right.$, Petroleum Ether-EtOAc, 400:1]. The enantiomeric excess $(93 \%$ ee $)$ was determined by chiral HPLC in comparison with the racemate (see below).

$[\alpha]_{\mathrm{D}}^{23}-53.8\left(\mathrm{c}=0.91, \mathrm{CHCl}_{3}\right)$.

IR (film): 1784, 1520, 1092, $1008 \mathrm{~cm}^{-1}$.

${ }^{1} \mathrm{H}$ NMR (400 MHz, $\left.\mathrm{CDCl}_{3}\right): \delta=7.40-7.19(\mathrm{~m}, 2 \mathrm{H}), 6.99-6.78(\mathrm{~m}, 2 \mathrm{H}), 5.98-5.55(\mathrm{~m}, 1 \mathrm{H})$, $5.23-4.98(\mathrm{~m}, 2 \mathrm{H}), 3.95(\mathrm{t}, J=7.7 \mathrm{~Hz}, 1 \mathrm{H}), 3.82(\mathrm{~s}, 3 \mathrm{H}), 2.97-2.84(\mathrm{~m}, 1 \mathrm{H}), 2.67-2.56(\mathrm{~m}, 1 \mathrm{H})$.

${ }^{13} \mathrm{C}$ NMR $\left(101 \mathrm{MHz}, \mathrm{CDCl}_{3}\right): \delta=169.7,159.2,134.1,128.9,128.7,117.8,114.3,77.3,77.0$, $76.7,55.2,50.0,37.4$. 
${ }^{19} \mathrm{~F}$ NMR (376 MHz, $\left.\mathrm{CDCl}_{3}\right): \delta=-149.05--154.71(\mathrm{~m}),-158.15,-161.23--165.15(\mathrm{~m})$.

HRMS (CI): $m / z$ calcd for $[\mathrm{M}+\mathrm{H}]^{+} \mathrm{C}_{18} \mathrm{H}_{14} \mathrm{~F}_{5} \mathrm{O}_{3}: 373.08$. Found: 373.08 .

HPLC analysis using a chiral column (Chiralpak IA $3 \mu$ column, $22{ }^{\circ} \mathrm{C}, 0.5 \mathrm{~mL} / \mathrm{min}, 200: 1$ Hexane:Isopropanol, $210 \mathrm{~nm}, \mathrm{t}_{\text {minor }}=10.9 \mathrm{~min}, \mathrm{t}_{\text {major }}=11.7 \mathrm{~min}$ ).

Pentafluorophenyl 2-(4-chlorophenyl)pent-4-enoate (9): Prepared according to General

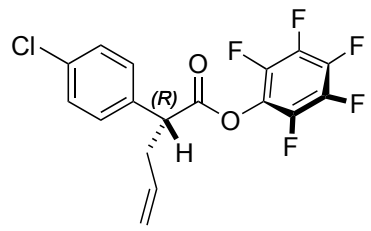
Procedure C. The title compound 9 was obtained $(34 \mathrm{mg}, 0.091 \mathrm{mmol}$, $91 \%$ ) as a colorless oil following purification by column chromatography $\left[\mathrm{SiO}_{2}\right.$, Petroleum Ether-EtOAc, 400:1]. The enantiomeric excess $(88 \%$ ee) was determined by chiral HPLC in comparison with the racemate (see below).

$[\alpha]_{\mathrm{D}}^{23}-68.9\left(\mathrm{c}=1.42, \mathrm{CHCl}_{3}\right)$.

IR (film): 1774, 1517, $1096 \mathrm{~cm}^{-1}$.

${ }^{1} \mathrm{H}$ NMR $\left(400 \mathrm{MHz}, \mathrm{CDCl}_{3}\right): \delta=7.36(\mathrm{dd}, J=8.6,1.7 \mathrm{~Hz}, 2 \mathrm{H}), 7.34-7.28(\mathrm{~m}, 2 \mathrm{H}), 5.93-5.64$ (m, 1H), 5.31-4.99 (m, 2H), 3.98 (ddd, $J=8.3,6.9,1.5 \mathrm{~Hz}, 1 \mathrm{H}), 2.95-2.89(\mathrm{~m}, 1 \mathrm{H}), 2.65-2.59$ $(\mathrm{m}, 1 \mathrm{H})$.

${ }^{13} \mathrm{C}$ NMR $\left(101 \mathrm{MHz}, \mathrm{CDCl}_{3}\right): \delta=169.1,135.1,134.0,133.6,129.3,129.1,118.3,77.3,77.0$, 76.7, 50.2, 37.3.

${ }^{19} \mathrm{~F}$ NMR (376 MHz, $\left.\mathrm{CDCl}_{3}\right): \delta=-149.05--154.71(\mathrm{~m}),-158.15,-161.23--165.15(\mathrm{~m})$.

HRMS (CI): $m / z$ calcd for $[\mathrm{M}+\mathrm{H}]^{+} \mathrm{C}_{17} \mathrm{H}_{11} \mathrm{~F}_{5} \mathrm{O}_{2} \mathrm{Cl}: 377.03$. Found: 377.03 .

HPLC analysis using a chiral column (Chiralpak IA $3 \mu$ column, $22{ }^{\circ} \mathrm{C}, 0.5 \mathrm{~mL} / \mathrm{min}, 200: 1$ Hexane:Isopropanol, $\left.210 \mathrm{~nm}, \mathrm{t}_{\text {minor }}=8.0 \mathrm{~min}, \mathrm{t}_{\text {major }}=10.0 \mathrm{~min}\right)$.

Pentafluorophenyl 2-(2-methylphenyl)pent-4-enoate (10): Prepared according to General

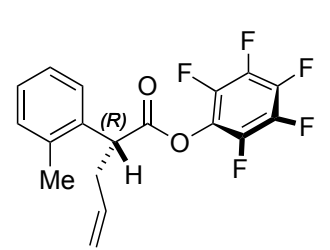
Procedure C. The title compound 10 was obtained $(32 \mathrm{mg}, 0.090 \mathrm{mmol}$, $90 \%$ ) as a colorless oil following purification by column chromatography $\left[\mathrm{SiO}_{2}\right.$, Petroleum Ether-EtOAc, 400:1]. The enantiomeric excess (91\% ee) was determined by chiral HPLC in comparison with the racemate (see below).

$[\alpha]_{\mathrm{D}}^{23}-92\left(\mathrm{c}=0.18, \mathrm{CHCl}_{3}\right)$.

IR (film): 1777, 1519, $1091 \mathrm{~cm}^{-1}$.

${ }^{1} \mathrm{H}$ NMR (500 MHz, $\left.\mathrm{CDCl}_{3}\right): \delta=7.38-7.34(\mathrm{~m}, 1 \mathrm{H}), 7.34-7.17(\mathrm{~m}, 3 \mathrm{H}), 5.83$ (ddt, $J=17.1$, $10.2,6.8 \mathrm{~Hz}, 1 \mathrm{H}), 5.35-4.93(\mathrm{~m}, 2 \mathrm{H}), 4.29(\mathrm{dd}, J=8.5,6.6 \mathrm{~Hz}, 1 \mathrm{H}), 3.07-2.84(\mathrm{~m}, 1 \mathrm{H}), 2.73-$ $2.58(\mathrm{~m}, 1 \mathrm{H}), 2.48(\mathrm{~s}, 3 \mathrm{H})$.

${ }^{13} \mathrm{C}$ NMR $\left(126 \mathrm{MHz}, \mathrm{CDCl}_{3}\right): \delta=170.04,156.96,134.92,129.16,129.02,125.70,120.84$, $117.52,110.73,77.41,77.36,77.16,76.91,55.47,45.13,35.73$. 
${ }^{19} \mathrm{~F}$ NMR (376 MHz, $\left.\mathrm{CDCl}_{3}\right): \delta=-151.37--153.77(\mathrm{~m}),-158.13(\mathrm{t}, J=21.7 \mathrm{~Hz}),-162.53(\mathrm{td}, J$ $=22.1,4.9 \mathrm{~Hz})$.

HRMS (CI): $m / z$ calcd for $[\mathrm{M}+\mathrm{H}]^{+} \mathrm{C}_{18} \mathrm{H}_{14} \mathrm{~F}_{5} \mathrm{O}_{2}$ : 357.09. Found: 357.09 .

HPLC analysis using a chiral column (Chiralpak IA $3 \mu$ column, $22{ }^{\circ} \mathrm{C}, 0.5 \mathrm{~mL} / \mathrm{min}, 200: 1$ Hexane:Isopropanol, $210 \mathrm{~nm}, \mathrm{t}_{\text {minor }}=6.8 \mathrm{~min}, \mathrm{t}_{\text {major }}=7.3 \mathrm{~min}$ ).

Pentafluorophenyl 2-(3,4-dimethoxy-2-bromophenyl)pent-4-enoate (11): Prepared according

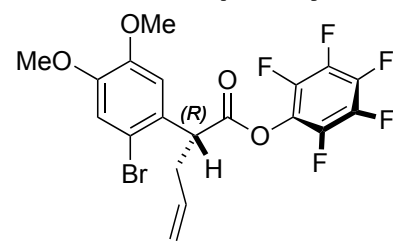
to General Procedure C. The title compound 11 was obtained $(32 \mathrm{mg}$, $0.067 \mathrm{mmol}, 67 \%$ ) as a colorless oil following purification by column chromatography $\left[\mathrm{SiO}_{2}\right.$, Petroleum Ether-EtOAc, 400:1]. The enantiomeric excess $(87 \%$ ee $)$ was determined by chiral HPLC in comparison with the racemate (see below).

$[\alpha]_{\mathrm{D}}^{23}-41.7\left(\mathrm{c}=0.68, \mathrm{CHCl}_{3}\right)$.

IR (film): 1785, 1524, 1090, $1006 \mathrm{~cm}^{-1}$.

${ }^{1} \mathrm{H}$ NMR $\left(500 \mathrm{MHz}, \mathrm{CDCl}_{3}\right): \delta=7.07(\mathrm{~s}, 1 \mathrm{H}), 6.86(\mathrm{~s}, 1 \mathrm{H}), 5.89-5.74(\mathrm{~m}, 1 \mathrm{H}), 5.26-5.01(\mathrm{~m}$, $2 \mathrm{H}), 4.55(\mathrm{dd}, J=8.3,6.7 \mathrm{~Hz}, 1 \mathrm{H}), 3.88(\mathrm{~s}, 3 \mathrm{H}), 3.87(\mathrm{~s}, 3 \mathrm{H}), 2.82-2.89(\mathrm{~m}, 1 \mathrm{H}), 2.68-2.54(\mathrm{~m}$, $1 \mathrm{H})$.

${ }^{13} \mathrm{C} \mathrm{NMR}\left(126 \mathrm{MHz}, \mathrm{CDCl}_{3}\right): \delta=169.5,149.3,149.0,133.8,128.3,118.3,115.8,114.9,110.8$, $77.4,77.2,76.9,56.3,56.2,49.3,37.1$.

${ }^{19} \mathrm{~F}$ NMR $\left(376 \mathrm{MHz}, \mathrm{CDCl}_{3}\right): \delta=-147.17--153.48(\mathrm{~m}),-157.76(\mathrm{t}, J=21.7 \mathrm{~Hz}),-162.24(\mathrm{td}, J$ $=22.6,5.1 \mathrm{~Hz})$.

HRMS (CI): $m / z$ calcd for $[\mathrm{M}]^{+} \mathrm{C}_{19} \mathrm{H}_{14} \mathrm{~F}_{5} \mathrm{O}_{4} \mathrm{Br}$ : 480.00. Found: 480.00 .

HPLC analysis using a chiral column (Chiralpak IA $3 \mu$ column, $22{ }^{\circ} \mathrm{C}, 0.5 \mathrm{~mL} / \mathrm{min}, 400: 1$ Hexane:Isopropanol, $210 \mathrm{~nm}, \mathrm{t}_{\text {minor }}=25.6 \mathrm{~min}, \mathrm{t}_{\text {major }}=29.8 \mathrm{~min}$ ).

Pentafluorophenyl 2-(4-isopropylphenyl)pent-4-enoate (12): Prepared according to General

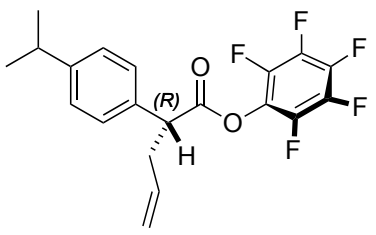
Procedure C. The title compound 12 was obtained $(29 \mathrm{mg}, 0.076 \mathrm{mmol}$, $76 \%$ as a colorless oil following purification by column chromatography $\left[\mathrm{SiO}_{2}\right.$, Petroleum Ether-EtOAc, 400:1]. The enantiomeric excess $(85 \%$ ee $)$ was determined by chiral HPLC in comparison with the racemate (see below).

$[\alpha]_{\mathrm{D}}^{23}-46.3\left(\mathrm{c}=0.5, \mathrm{CHCl}_{3}\right)$.

IR (film): 2962, 1784, 1521, $1092 \mathrm{~cm}^{-1}$.

${ }^{1} \mathrm{H}$ NMR (400 MHz, $\left.\mathrm{CDCl}_{3}\right): \delta=7.32-7.18(\mathrm{~m}, 4 \mathrm{H}), 5.80(\mathrm{ddt}, J=17.1,10.2,6.8 \mathrm{~Hz}, 1 \mathrm{H})$, $5.23-5.03(\mathrm{~m}, 2 \mathrm{H}), 3.98(\mathrm{dd}, J=8.9,6.5 \mathrm{~Hz}, 1 \mathrm{H}), 2.97-2.84(\mathrm{~m}, 2 \mathrm{H}), 2.69-2.56(\mathrm{~m}, 1 \mathrm{H})$, $1.25(\mathrm{~d}, J=6.9 \mathrm{~Hz}, 6 \mathrm{H})$.

${ }^{13} \mathrm{C}$ NMR (126 MHz, $\left.\mathrm{CDCl}_{3}\right): \delta=169.60,148.63,139.87,134.27,134.03,127.74,126.97$, $117.78,77.21,50.58,37.46,33.75,28.14,23.91,23.89$. 
${ }^{19} \mathrm{~F}$ NMR $\left(376 \mathrm{MHz}, \mathrm{CDCl}_{3}\right): \delta=-152.73(\mathrm{~d}, J=17.7 \mathrm{~Hz}),-158.78(\mathrm{t}, J=21.6 \mathrm{~Hz}),-163.12(\mathrm{t}, J$ $=19.6 \mathrm{~Hz})$.

HRMS (CI): $m / z$ calcd for $[\mathrm{M}+\mathrm{H}]^{+} \mathrm{C}_{20} \mathrm{H}_{18} \mathrm{~F}_{5} \mathrm{O}_{2}: 385.12$. Found: 385.12 .

HPLC analysis using a chiral column (Chiralpak IA $3 \mu$ column, $22{ }^{\circ} \mathrm{C}, 0.5 \mathrm{~mL} / \mathrm{min}, 400: 1$ Hexane:Isopropanol, $\left.210 \mathrm{~nm}, \mathrm{t}_{\text {minor }}=26.1 \mathrm{~min}, \mathrm{t}_{\text {major }}=30.6 \mathrm{~min}\right)$.

Pentafluorophenyl 2-(2-methoxyphenyl)pent-4-enoate (13): Prepared according to General

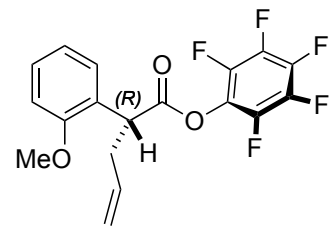
Procedure C. The title compound 13 was obtained $(34 \mathrm{mg}, 0.082 \mathrm{mmol}$, $82 \%$ ) as a colorless oil following purification by column chromatography $\left[\mathrm{SiO}_{2}\right.$, Petroleum Ether-EtOAc, 400:1]. The enantiomeric excess (92\% ee) was determined by chiral HPLC in comparison with the racemate (see below).

$[\alpha]_{\mathrm{D}}^{23}-61.5\left(\mathrm{c}=0.69, \mathrm{CHCl}_{3}\right)$.

IR (film): 1784, 1520, 1092, $1008 \mathrm{~cm}^{-1}$

${ }^{1} \mathrm{H}$ NMR $\left(400 \mathrm{MHz}, \mathrm{CDCl}_{3}\right): \delta=7.41-7.20(\mathrm{~m}, 2 \mathrm{H}), 7.01-6.83(\mathrm{~m}, 2 \mathrm{H}), 5.78$ (tdd, $J=16.4$, 8.6, $3.9 \mathrm{~Hz}, 1 \mathrm{H}), 5.17-4.96(\mathrm{~m}, 2 \mathrm{H}), 4.27(\mathrm{t}, J=7.4 \mathrm{~Hz}, 1 \mathrm{H}), 3.84(\mathrm{~s}, 3 \mathrm{H}), 2.97-2.81(\mathrm{~m}, 1 \mathrm{H})$, $2.74-2.49(\mathrm{~m}, 1 \mathrm{H})$.

${ }^{13} \mathrm{C}$ NMR $\left(101 \mathrm{MHz} \mathrm{CDCl}_{3}\right): \delta=170.0,156.9,134.9,129.2,129.0,125.7,120.8,117.5,77.5$, 77.2, 76.8, 55.5, 45.1, 35.7.

${ }^{19} \mathrm{~F}$ NMR $\left(376 \mathrm{MHz}, \mathrm{CDCl}_{3}\right): \delta=-150.36--154.49(\mathrm{~m}),-158.46(\mathrm{t}, J=21.7 \mathrm{~Hz}),-162.76(\mathrm{td}, J$ $=22.4,5.1 \mathrm{~Hz})$.

HRMS (CI): $m / z$ calcd for $[\mathrm{M}+\mathrm{H}]^{+} \mathrm{C}_{18} \mathrm{H}_{14} \mathrm{~F}_{5} \mathrm{O}_{3}: 373.08$. Found: 373.08 .

HPLC analysis using a chiral column (Chiralpak IA $3 \mu$ column, $22{ }^{\circ} \mathrm{C}, 0.5 \mathrm{~mL} / \mathrm{min}, 200: 1$ Hexane:Isopropanol, $\left.210 \mathrm{~nm}, \mathrm{t}_{\text {minor }}=9.1 \mathrm{~min}, \mathrm{t}_{\text {major }}=8.5 \mathrm{~min}\right)$.

Pentafluorophenyl 2-(naphthalene-2-yl)pent-4-enoate (14): Prepared according to General

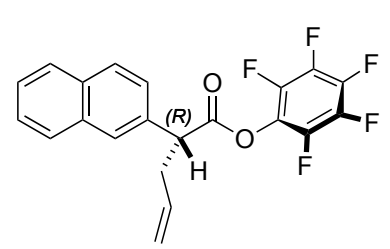
Procedure C. The title compound 14 was obtained $(33 \mathrm{mg}, 0.086 \mathrm{mmol}$, $86 \%$ as a colorless oil following purification by column chromatography $\left[\mathrm{SiO}_{2}\right.$, Petroleum Ether-EtOAc, 400:1]. The enantiomeric excess $(85 \%$ ee $)$ was determined by chiral HPLC in comparison with the racemate (see below).

$[\alpha]_{\mathrm{D}}^{23}-26\left(\mathrm{c}=0.03, \mathrm{CHCl}_{3}\right)$.

IR (film): 1782, 1521, 1090, $999 \mathrm{~cm}^{-1}$.

${ }^{1} \mathrm{H}$ NMR $\left(400 \mathrm{MHz}, \mathrm{CDCl}_{3}\right): \delta=7.92-7.81(\mathrm{~m}, 4 \mathrm{H}), 7.56-7.46(\mathrm{~m}, 3 \mathrm{H}), 5.83(\mathrm{~m}, 1 \mathrm{H}), 5.19$ (dd, $J=17.1,1.6 \mathrm{~Hz}, 1 \mathrm{H}), 5.11(\mathrm{dd}, J=10.2,1.4 \mathrm{~Hz}, 1 \mathrm{H}), 4.23-4.11(\mathrm{~m}, 1 \mathrm{H}), 3.11-2.98(\mathrm{~m}, 1 \mathrm{H})$, $2.82-2.69(\mathrm{~m}, 1 \mathrm{H})$. 
${ }^{13} \mathrm{C}$ NMR (101 MHz, $\left.\mathrm{CDCl}_{3}\right): \delta=169.78,134.44,134.35,133.74,133.21,129.11,128.21$, $128.03,127.48,126.78,126.62,125.75,118.36,77.65,77.33,77.01,51.38,37.71$.

${ }^{19} \mathrm{~F}$ NMR (376 MHz, $\left.\mathrm{CDCl}_{3}\right): \delta=-151.97--153.47(\mathrm{~m}),-157.96(\mathrm{t}, J=21.6 \mathrm{~Hz}),-162.22--$ $163.05(\mathrm{~m})$.

HRMS (CI): $m / z$ calcd for $[\mathrm{M}+\mathrm{H}]^{+} \mathrm{C}_{21} \mathrm{H}_{14} \mathrm{~F}_{5} \mathrm{O}_{3}: 393.09$. Found: 393.09 .

HPLC analysis using a chiral column (Chiralpak IB $5 \mu$ column, $22{ }^{\circ} \mathrm{C}, 0.5 \mathrm{~mL} / \mathrm{min}, 1200: 1$ Hexane:Isopropanol, $\left.210 \mathrm{~nm}, \mathrm{t}_{\text {minor }}=23.1 \mathrm{~min}, \mathrm{t}_{\mathrm{major}}=21.2 \mathrm{~min}\right)$.

Pentafluorophenyl 2-(2,3,4-trifluorophenyl)pent-4-enoate (15): Prepared according to General

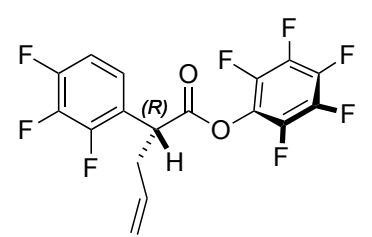
Procedure C. The title compound 15 was obtained $(32 \mathrm{mg}, 0.080 \mathrm{mmol}$, $80 \%$ ) as a colorless oil following purification by column chromatography $\left[\mathrm{SiO}_{2}\right.$, Petroleum Ether-EtOAc, 400:1]. The enantiomeric excess $(87 \%$ $e e$ ) was determined by chiral HPLC in comparison with the racemate (see below).

$[\alpha]_{\mathrm{D}}^{23}-75.0\left(\mathrm{c}=1.9, \mathrm{CHCl}_{3}\right)$.

IR (film): 1770, 1522, 1096, $1008 \mathrm{~cm}^{-1}$.

${ }^{1} \mathrm{H}$ NMR (400 MHz, $\left.\mathrm{CDCl}_{3}\right): \delta=7.41-7.17(\mathrm{~m}, 1 \mathrm{H}), 7.17-7.06(\mathrm{~m}, 1 \mathrm{H}), 7.06-6.94(\mathrm{~m}, 1 \mathrm{H})$, 6.50-6.41 (m, 1H), 6.16-6.04 (m, 1H), $4.38(\mathrm{t}, J=7.5 \mathrm{~Hz}, 1 \mathrm{H}), 3.13-3.01(\mathrm{~m}, 1 \mathrm{H}), 2.81-2.68$ $(\mathrm{m}, 1 \mathrm{H})$.

${ }^{13} \mathrm{C}$ NMR (100 MHz, $\left.\mathrm{CDCl}_{3}\right): \delta=168.24,149.83,141.10,140.68,140.04,139.08,136.67$, $133.97,129.00,128.58,128.06,127.68,126.65,126.22,124.26,122.56,122.52,122.49,121.67$, $112.69,112.66,112.55,112.52,77.21,43.35,35.64,27.80$.

${ }^{19} \mathrm{~F}$ NMR (376 MHz, $\left.\mathrm{CDCl}_{3}\right): \delta=-133.94$ (dtd, $\left.J=20.5,8.7,5.3 \mathrm{~Hz}\right),-137.35(\mathrm{~d}, J=19.4 \mathrm{~Hz})$, $151.71--153.11(\mathrm{~m}),-157.29(\mathrm{t}, J=21.8 \mathrm{~Hz}),-158.56--160.27(\mathrm{~m}),-161.35--163.22(\mathrm{~m})$.

HRMS (CI): $m / z$ calcd for $[\mathrm{M}-\mathrm{H}] \mathrm{C}_{17} \mathrm{H}_{7} \mathrm{~F}_{8} \mathrm{O}_{2}$ : 395.03. Found: 395.03.

HPLC analysis using a chiral column (Chiralpak IA $3 \mu$ column, $22{ }^{\circ} \mathrm{C}, 0.5 \mathrm{~mL} / \mathrm{min}, 400: 1$ Hexane:Isopropanol, $\left.210 \mathrm{~nm}, \mathrm{t}_{\text {minor }}=9.4 \mathrm{~min}, \mathrm{t}_{\text {major }}=11.4 \mathrm{~min}\right)$.

Pentafluorophenyl 2-(4-vinylphenyl)pent-4-enoate (16): Prepared according to General

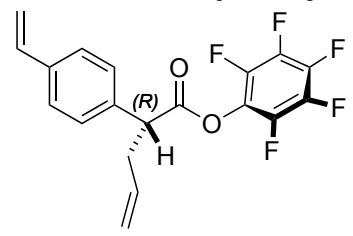
Procedure C. The title compound 16 was obtained $(29 \mathrm{mg}, 0.079 \mathrm{mmol}$, $79 \%$ ) as a colorless oil following purification by column chromatography $\left[\mathrm{SiO}_{2}\right.$, Petroleum Ether-EtOAc, 400:1]. The enantiomeric excess (92\% ee) was determined by chiral HPLC in comparison with the racemate (see below).

$[\alpha]_{\mathrm{D}}^{23}-90\left(\mathrm{c}=0.17, \mathrm{CHCl}_{3}\right)$.

IR (film): 1792, 1520, 1112, $1005 \mathrm{~cm}^{-1}$. 
${ }^{1} \mathrm{H}$ NMR $\left(400 \mathrm{MHz}, \mathrm{CDCl}_{3}\right): \delta=7.39(\mathrm{~d}, J=2.0 \mathrm{~Hz}, 1 \mathrm{H}), 7.36-7.28(\mathrm{~m}, 1 \mathrm{H}), 6.73-6.64(\mathrm{~m}$, $1 \mathrm{H}), 5.84-5.70(\mathrm{~m}, 2 \mathrm{H}), 5.25(\mathrm{dd}, J=10.9,0.9 \mathrm{~Hz}, 1 \mathrm{H}), 5.17-5.07(\mathrm{~m}, 1 \mathrm{H}), 3.97(\mathrm{dd}, J=8.5$, $6.9 \mathrm{~Hz}, 1 \mathrm{H}), 2.91(\mathrm{ddd}, J=14.4,8.4,7.1 \mathrm{~Hz}, 1 \mathrm{H}), 2.62(\mathrm{dt}, J=14.1,6.6 \mathrm{~Hz}, 1 \mathrm{H})$.

${ }^{13} \mathrm{C}$ NMR $\left(126 \mathrm{MHz}, \mathrm{CDCl}_{3}\right): \delta=169.38,137.35,136.18,133.99,132.13,128.10,126.74$, $118.00,114.40,77.21,50.64,37.31,29.71,14.31$.

${ }^{19} \mathrm{~F}$ NMR $\left(376 \mathrm{MHz}, \mathrm{CDCl}_{3}\right): \delta=-152.09--152.93(\mathrm{~m}),-157.97(\mathrm{t}, J=21.7 \mathrm{~Hz}),-161.98--$ $163.11(\mathrm{~m})$.

HRMS (CI): $m / z$ calcd for $[\mathrm{M}+\mathrm{H}] \mathrm{C}_{19} \mathrm{H}_{14} \mathrm{~F}_{5} \mathrm{O}_{2}$ : 369.09. Found: 369.09 .

HPLC analysis using a chiral column (Chiralpak IA $3 \mu$ column, $22{ }^{\circ} \mathrm{C}, 0.5 \mathrm{~mL} / \mathrm{min}, 400: 1$ Hexane:Isopropanol, $210 \mathrm{~nm}, \mathrm{t}_{\text {minor }}=10.4 \mathrm{~min}, \mathrm{t}_{\text {major }}=11.5 \mathrm{~min}$ ).

\section{Pentafluorophenyl 2-(1-(4-chlorobenzoyl)-5-methoxy-1H-indol-2-yl)pent-4-enoate (17):}

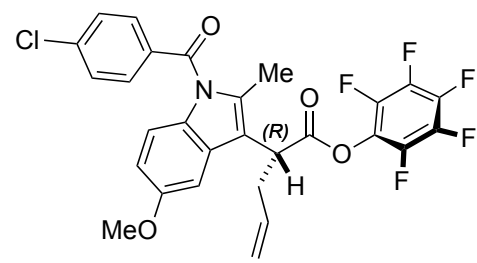

Prepared according to General Procedure C. The title compound 17 was obtained (34 mg, $0.06 \mathrm{mmol}, 60 \%$ ) as a yellow oil following purification by column chromatography $\left[\mathrm{SiO}_{2}\right.$, Petroleum Ether-EtOAc, 400:1]. The enantiomeric excess (95\% ee) was determined by chiral HPLC in comparison with the racemate (see below).

$[\alpha]_{\mathrm{D}}^{23}-54.0\left(\mathrm{c}=0.5, \mathrm{CHCl}_{3}\right)$.

IR (film): 2931, 1782, 1686, 1590, $1522 \mathrm{~cm}^{-1}$.

${ }^{1} \mathrm{H}$ NMR (400 MHz, $\left.\mathrm{CDCl}_{3}\right): \delta=7.74-7.62(\mathrm{~m}, 2 \mathrm{H}), 7.49-7.45(\mathrm{~m}, 2 \mathrm{H}), 7.05(\mathrm{~d}, J=2.5 \mathrm{~Hz}$, $1 \mathrm{H}), 6.89(\mathrm{~d}, J=9.0 \mathrm{~Hz}, 1 \mathrm{H}), 6.70(\mathrm{dd}, J=9.1,2.5 \mathrm{~Hz}, 1 \mathrm{H}), 5.77$ (ddt, $J=17.1,10.1,6.9 \mathrm{~Hz}$, $1 \mathrm{H}), 5.21-5.06(\mathrm{~m}, 2 \mathrm{H}), 4.24-4.13(\mathrm{~m}, 1 \mathrm{H}), 3.83(\mathrm{~s}, 3 \mathrm{H}), 3.11(\mathrm{dt}, J=14.2,7.1 \mathrm{~Hz}, 1 \mathrm{H}), 2.78$ $(\mathrm{dt}, J=14.8,7.7 \mathrm{~Hz}, 1 \mathrm{H}), 2.43(\mathrm{~s}, 3 \mathrm{H})$.

${ }^{13} \mathrm{C}$ NMR $\left(100 \mathrm{MHz}, \mathrm{CDCl}_{3}\right): \delta=169.03,168.28,155.83,139.59,135.90,134.16,133.60$, $131.25,130.98,129.18,128.74,117.96,114.85,114.29,111.80,101.85,77.17,55.65,41.73$, $34.66,13.33$.

${ }^{19} \mathrm{~F} \mathrm{NMR}\left(376 \mathrm{MHz}, \mathrm{CDCl}_{3}\right): \delta=-150.62--153.67(\mathrm{~m}),-157.76(\mathrm{t}, J=21.7 \mathrm{~Hz}),-162.20(\mathrm{dd}, J$ $=21.8,17.3 \mathrm{~Hz}$ ).

HRMS (CI): $m / z$ calcd for $[\mathrm{M}]^{+} \mathrm{C}_{28} \mathrm{H}_{19} \mathrm{~F}_{5} \mathrm{ClNO}_{5}$ : 563.09. Found: 563.09 .

HPLC analysis using a chiral column (Chiralpak IA $5 \mu$ column, $22{ }^{\circ} \mathrm{C}, 0.5 \mathrm{~mL} / \mathrm{min}, 99: 1$ Hexane:Isopropanol, $210 \mathrm{~nm}, \mathrm{t}_{\text {minor }}=16.2 \mathrm{~min}, \mathrm{t}_{\text {major }}=22.4 \mathrm{~min}$ ). 
Pentafluorophenyl (E)-2,5-diphenylpent-4-enoate (18): Prepared according to General

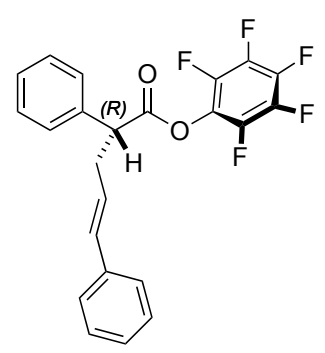

Procedure C. The title compound 18 was obtained $(36 \mathrm{mg}, 0.085 \mathrm{mmol}$, $85 \%$ ) as a white solid following purification by column chromatography $\left[\mathrm{SiO}_{2}\right.$, Petroleum Ether-EtOAc, 400:1]. The enantiomeric excess (93\% ee) was determined by chiral HPLC in comparison with the racemate (see below).

M.p. $($ pentane-EtOAc, $400: 1$; needles $)=107-108{ }^{\circ} \mathrm{C}$

$[\alpha]_{\mathrm{D}}^{23}-110\left(\mathrm{c}=0.175, \mathrm{CHCl}_{3}\right)$.

IR (film): 1004, 1105, 1525, $1780 \mathrm{~cm}^{-1}$.

${ }^{1} \mathrm{H}$ NMR (500 MHz, $\left.\mathrm{CDCl}_{3}\right): \delta=7.43-7.39(\mathrm{~m}, 4 \mathrm{H}), 7.35(\mathrm{dd}, J=4.8,3.7 \mathrm{~Hz}, 1 \mathrm{H}), 7.34-7.28$ $(\mathrm{m}, 4 \mathrm{H}), 7.23(\mathrm{~d}, J=6.5 \mathrm{~Hz}, 1 \mathrm{H}), 6.51(\mathrm{dd}, J=15.8,1.4 \mathrm{~Hz}, 1 \mathrm{H}), 6.17(\mathrm{dt}, J=15.7,7.1 \mathrm{~Hz}, 1 \mathrm{H})$, $4.07(\mathrm{dd}, J=8.6,6.7 \mathrm{~Hz}, 1 \mathrm{H}), 3.17-3.00(\mathrm{~m}, 1 \mathrm{H}), 2.88-2.65(\mathrm{~m}, 1 \mathrm{H})$.

${ }^{13} \mathrm{C} \mathrm{NMR}\left(126 \mathrm{MHz}, \mathrm{CDCl}_{3}\right): \delta=169.63,137.17,136.88,133.30,129.16,128.68,128.21$, $128.07,127.60,126.33,125.66,77.41,77.36,77.16,76.91,51.49,36.93$.

${ }^{19} \mathrm{~F}$ NMR (376 MHz, $\left.\mathrm{CDCl}_{3}\right): \delta=-149.42--154.06(\mathrm{~m}),-157.96(\mathrm{t}, J=21.7 \mathrm{~Hz}),-161.16--$ $165.80(\mathrm{~m})$.

HRMS (CI): $m / z$ calcd for $[\mathrm{M}]^{+} \mathrm{C}_{23} \mathrm{H}_{15} \mathrm{~F}_{5} \mathrm{O}_{2}: 418.09$. Found: 418.09.

HPLC analysis using a chiral column (Chiralpak IA $3 \mu$ column, $22{ }^{\circ} \mathrm{C}, 0.5 \mathrm{~mL} / \mathrm{min}, 400: 1$ Hexane:Isopropanol, $210 \mathrm{~nm}, \mathrm{t}_{\text {minor }}=13.6 \mathrm{~min}, \mathrm{t}_{\text {major }}=14.2 \mathrm{~min}$ ).

Pentafluorophenyl (E)-2-phenyl-5-(4-(methoxy)phenyl)pent-4-enoate (19): Prepared according

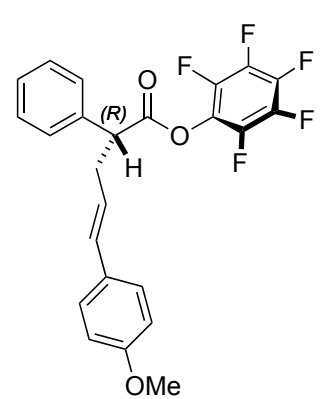
to General Procedure C. The title compound 19 was obtained (41 mg, 0.091 mmol, 91\%) as a white solid following purification by column chromatography [ $\mathrm{SiO}_{2}$, Petroleum Ether-EtOAc, 400:1]. The enantiomeric excess $(93 \%$ ee) was determined by chiral HPLC in comparison with the racemate (see below).

M.p. (pentane-EtOAc, 400:1; needles $)=130-132{ }^{\circ} \mathrm{C}$

$[\alpha]_{\mathrm{D}}-30.5\left(\mathrm{c}=070, \mathrm{CHCl}_{3}\right)$.

IR (film): 1004, 1105, 1525, $1780 \mathrm{~cm}^{-1}$.

${ }^{1} \mathrm{H}$ NMR $\left(400 \mathrm{MHz}, \mathrm{CDCl}_{3}\right): \delta=7.40(\mathrm{~d}, J=4.3 \mathrm{~Hz}, 4 \mathrm{H}), 7.38-7.33(\mathrm{~m}, 1 \mathrm{H}), 7.24(\mathrm{~s}, 2 \mathrm{H}), 6.84$ $(\mathrm{d}, J=8.6 \mathrm{~Hz}, 2 \mathrm{H}), 6.45(\mathrm{~d}, J=15.8 \mathrm{~Hz}, 1 \mathrm{H}), 6.02(\mathrm{dt}, J=15.8,7.1 \mathrm{~Hz}, 1 \mathrm{H}), 4.06(\mathrm{dd}, J=8.6$, $6.6 \mathrm{~Hz}, 1 \mathrm{H}), 3.80(\mathrm{~s}, 3 \mathrm{H}), 3.08(\mathrm{dt}, J=15.3,8.0 \mathrm{~Hz}, 1 \mathrm{H}), 2.78(\mathrm{dt}, J=13.9,6.7 \mathrm{~Hz}, 1 \mathrm{H})$.

${ }^{13} \mathrm{C} \mathrm{NMR}\left(101 \mathrm{MHz}, \mathrm{CDCl}_{3}\right): \delta=169.7,159.4,137.0,132.7,130.0,129.1,128.2,128.1,127.5$, $123.4,114.1,77.5,77.4,77.2,76.8,55.4,51.6,37.0$.

${ }^{19} \mathrm{~F}$ NMR (376 MHz, $\left.\mathrm{CDCl}_{3}\right): \delta=-149.63--154.42(\mathrm{~m}),-158.01(\mathrm{t}, J=21.7 \mathrm{~Hz}),-162.38(\mathrm{td}, J$ $=22.3,5.0 \mathrm{~Hz})$.

HRMS (CI): $m / z$ calcd for $[\mathrm{M}+\mathrm{H}]^{+} \mathrm{C}_{24} \mathrm{H}_{18} \mathrm{~F}_{5} \mathrm{O}_{3}: 449.11$. Found: 449.11. 
HPLC analysis using a chiral column (Chiralpak IA $3 \mu$ column, $22{ }^{\circ} \mathrm{C}, 0.5 \mathrm{~mL} / \mathrm{min}, 200: 1$ Hexane:Isopropanol, $210 \mathrm{~nm}, \mathrm{t}_{\text {minor }}=17.95 \mathrm{~min}, \mathrm{t}_{\text {major }}=21.3 \mathrm{~min}$ ).

Pentafluorophenyl (E)-2-phenyl-5-(4-(trifluoromethyl)phenyl)pent-4-enoate (20): Prepared

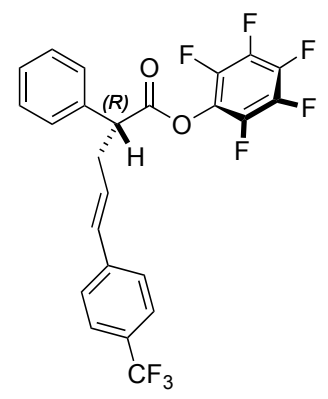
according to General Procedure C. The title compound 20 was obtained (42 $\mathrm{mg}, 0.088 \mathrm{mmol}, 88 \%$ ) as a white solid following purification by column chromatography [ $\mathrm{SiO}_{2}$, Petroleum Ether-EtOAc, 400:1]. The enantiomeric excess $(94 \%$ ee) was determined by chiral HPLC in comparison with the racemate (see below).

M.p. (pentane-EtOAc, 400:1; needles) $=125-127{ }^{\circ} \mathrm{C}$

$[\alpha]_{\mathrm{D}}^{23}-46.4\left(\mathrm{c}=1.34, \mathrm{CHCl}_{3}\right)$.

IR (film): 1004, 1115, 1525, $1780 \mathrm{~cm}^{-1}$.

${ }^{1} \mathrm{H}$ NMR $\left(400 \mathrm{MHz}, \mathrm{CDCl}_{3}\right): \delta=7.56(\mathrm{~s}, 1 \mathrm{H}), 7.54(\mathrm{~s}, 1 \mathrm{H}), 7.46-7.37(\mathrm{~m}, 7 \mathrm{H}), 6.54(\mathrm{~d}, J=15.8$ $\mathrm{Hz}, 1 \mathrm{H}), 6.27$ (dt, $J=15.8,7.1 \mathrm{~Hz}, 1 \mathrm{H}), 4.10$ (dd, $J=8.6,6.6 \mathrm{~Hz}, 1 \mathrm{H}), 3.26-3.04$ (m, 1H), 2.93$2.77(\mathrm{~m}, 1 \mathrm{H})$.

${ }^{13} \mathrm{C}$ NMR (101 MHz, $\left.\mathrm{CDCl}_{3}\right): \delta=169.5,140.6,136.7,132.1,129.3,129.2,128.5,128.3,128.0$, $126.5,125.7,125.6,77.5,77.2,76.8,51.2,36.9$.

${ }^{19} \mathrm{~F}$ NMR (376 MHz, $\left.\mathrm{CDCl}_{3}\right): \delta=-62.56,-150.29--154.49(\mathrm{~m}),-157.76(\mathrm{t}, J=21.6 \mathrm{~Hz})$, $162.23(\mathrm{dd}, J=21.9,17.3 \mathrm{~Hz})$.

HRMS (CI): $m / z$ calcd for $[\mathrm{M}+\mathrm{H}]{ }^{+} \mathrm{C}_{24} \mathrm{H}_{15} \mathrm{~F}_{8} \mathrm{O}_{2}: 487.09$. Found: 487.09.

HPLC analysis using a chiral column (Chiralpak IA $3 \mu$ column, $22{ }^{\circ} \mathrm{C}, 0.5 \mathrm{~mL} / \mathrm{min}, 200: 1$ Hexane:Isopropanol, $210 \mathrm{~nm}, \mathrm{t}_{\text {minor }}=16.5 \mathrm{~min}$, $\left.\mathrm{t}_{\text {major }}=18.0 \mathrm{~min}\right)$.

Pentafluorophenyl (E)-5-(3-chloro-4-methoxyphenyl)-2-phenylpent-4-enoate (21): Prepared

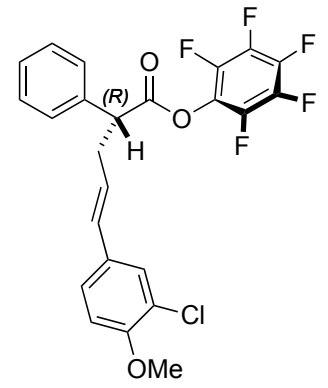
according to General Procedure C. The title compound 21 was obtained (41 $\mathrm{mg}, 0.085 \mathrm{mmol}, 85 \%$ ) as a white solid following purification by column chromatography $\left[\mathrm{SiO}_{2}\right.$, Petroleum Ether-EtOAc, 400:1]. The enantiomeric excess $(91 \%$ ee) was determined by chiral HPLC in comparison with the racemate (see below).

M.p. (pentane-EtOAc, 400:1; needles) $=113-115^{\circ} \mathrm{C}$

$[\alpha]_{\mathrm{D}}^{23}-43.7\left(\mathrm{c}=1.1, \mathrm{CHCl}_{3}\right)$.

IR (film): 1004, 1108, 1526, $1781 \mathrm{~cm}^{-1}$.

${ }^{1} \mathrm{H}$ NMR (400 MHz, $\left.\mathrm{CDCl}_{3}\right): \delta=7.44-7.37(\mathrm{~m}, 4 \mathrm{H}), 7.37-7.30(\mathrm{~m}, 2 \mathrm{H}), 7.15(\mathrm{dt}, J=8.5,2.1$ $\mathrm{Hz}, 1 \mathrm{H}), 6.85$ (dd, $J=8.6,1.8 \mathrm{~Hz}, 1 \mathrm{H}), 6.39$ (dd, $J=15.8,1.8 \mathrm{~Hz}, 1 \mathrm{H}), 6.04$ (dtd, $J=15.8,7.1$, $1.8 \mathrm{~Hz}, 1 \mathrm{H}), 4.06(\mathrm{ddd}, J=8.5,6.6,1.9 \mathrm{~Hz}, 1 \mathrm{H}), 3.89$ (s, 3H), 3.20-2.99 (m, 1H), 2.86-2.65 (m, $1 \mathrm{H})$. 
${ }^{13} \mathrm{C}$ NMR (101 MHz, $\left.\mathrm{CDCl}_{3}\right): \delta=169.59,154.46,136.80,131.56,130.91,129.17,128.23$, $128.03,127.83,125.82,124.89,122.74,112.10,77.48,77.16,76.84,56.34,51.45,36.86$.

$\left.{ }^{19} \mathrm{~F} \mathrm{NMR} \mathrm{(376} \mathrm{MHz,} \mathrm{CDCl}_{3}\right): \delta=-149.05--154.27(\mathrm{~m}),-157.91(\mathrm{t}, J=21.6 \mathrm{~Hz}),-162.31(\mathrm{td}, J$ $=23.3,5.4 \mathrm{~Hz})$.

HRMS (CI): $m / z$ calcd for $[\mathrm{M}+\mathrm{H}]^{+} \mathrm{C}_{24} \mathrm{H}_{17} \mathrm{~F}_{5} \mathrm{ClO}_{3}$ : 483.07. Found: 483.07.

HPLC analysis using a chiral column (Chiralpak IA $3 \mu$ column, $22{ }^{\circ} \mathrm{C}, 0.5 \mathrm{~mL} / \mathrm{min}, 200: 1$ Hexane:Isopropanol, $210 \mathrm{~nm}, \mathrm{t}_{\text {minor }}=26.5 \mathrm{~min}, \mathrm{t}_{\text {major }}=28.3 \mathrm{~min}$ ).

Pentafluorophenyl (E)-5-(4-((tert-butyldimethylsilyl)oxy)-3,5-dimethoxyphenyl)-2phenylpent-4-enoate (22): Prepared according to General Procedure C. The title compound 22 ( $F$ was obtained $(47 \mathrm{mg}, 0.070 \mathrm{mmol}, 70 \%)$ as colorless oil following 作 $\begin{aligned} & \text { purification by column chromatography }\left[\mathrm{SiO}_{2} \text {, Petroleum Ether-EtOAc, }\right. \\ & 400: 1] \text {. The enantiomeric excess }(91 \% \text { ee }) \text { was determined by chiral HPLC }\end{aligned}$ in comparison with the racemate (see below).

$[\alpha]_{\mathrm{D}}^{23}-19\left(\mathrm{c}=0.47, \mathrm{CHCl}_{3}\right)$.

IR (film): 697, 806, 838, 905, 996, 1129, 1247, 1334, 1464, 1518, 1581, $1780,2855,2929 \mathrm{~cm}^{-1}$.

${ }^{1} \mathrm{H}$ NMR $\left(400 \mathrm{MHz}, \mathrm{CDCl}_{3}\right): \delta=7.41(\mathrm{~m}, 4 \mathrm{H}), 7.35(\mathrm{q}, J=2.5,1.8 \mathrm{~Hz}, 1 \mathrm{H}), 6.52(\mathrm{~d}, J=1.8 \mathrm{~Hz}$, $2 \mathrm{H}), 6.43(\mathrm{~d}, J=15.7 \mathrm{~Hz}, 1 \mathrm{H}), 6.05(\mathrm{dt}, J=15.1,7.1 \mathrm{~Hz}, 1 \mathrm{H}), 4.11-4.05(\mathrm{~m}, 1 \mathrm{H}), 3.79(\mathrm{~s}, 6 \mathrm{H})$, 3.09 (dt, $J=15.6,8.2 \mathrm{~Hz}, 1 \mathrm{H}), 2.76$ (dt, $J=13.8,6.6 \mathrm{~Hz}, 1 \mathrm{H}), 1.00$ (s, 9H), 0.12 (s, 6H).

$\left.{ }^{13} \mathrm{C} \mathrm{NMR} \mathrm{(126} \mathrm{MHz,} \mathrm{CDCl}_{3}\right): \delta=169.73,151.78,137.00,134.47,133.47,129.93,129.16$, $129.00,128.20,128.16,128.01,123.84,103.63,77.41,77.36,77.16,76.91,55.99,55.87,51.67$, $37.01,29.86,25.95,25.83,18.89,-4.49$.

${ }^{19} \mathrm{~F} \mathrm{NMR}\left(376 \mathrm{MHz}, \mathrm{CDCl}_{3}\right): \delta=-152.38(\mathrm{~d}, J=19.5 \mathrm{~Hz}),-157.89,-162.34(\mathrm{~d}, J=21.4 \mathrm{~Hz})$.

HRMS (CI): $m / z$ calcd for $[\mathrm{M}+\mathrm{H}]^{+} \mathrm{C}_{31} \mathrm{H}_{34} \mathrm{~F}_{5} \mathrm{O}_{5} \mathrm{Si}$ : 609.20. Found: 609.20.

HPLC analysis using a chiral column (Chiralpak IA $3 \mu$ column, $22{ }^{\circ} \mathrm{C}, 0.5 \mathrm{~mL} / \mathrm{min}, 200: 1$ Hexane:Isopropanol, $210 \mathrm{~nm}, \mathrm{t}_{\text {minor }}=20.2 \mathrm{~min}, \mathrm{t}_{\text {major }}=22.2 \mathrm{~min}$ ).

Pentafluorophenyl E)-5-(naphthalen-2-yl)-2-phenylpent-4-enoate (23): Prepared according to

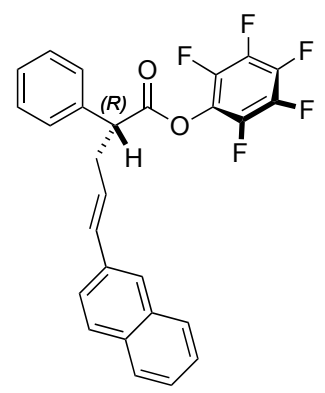
General Procedure C. The title compound 23 was obtained (41 mg, 0.087 mmol, 87\%) as a white solid following purification by column chromatography [ $\mathrm{SiO}_{2}$, Petroleum Ether-EtOAc, 400:1]. The enantiomeric excess $(93 \%$ ee) was determined by chiral HPLC in comparison with the racemate (see below).

M.p. (pentane-EtOAc, 400:1; needles $)=137-139^{\circ} \mathrm{C}$ 
$[\alpha]_{\mathrm{D}}^{23}-48.3\left(\mathrm{c}=0.88, \mathrm{CHCl}_{3}\right)$.

IR (film): 1004, 1105, 1525, $1780 \mathrm{~cm}^{-1}$.

${ }^{1} \mathrm{H}$ NMR (400 MHz, $\left.\mathrm{CDCl}_{3}\right): \delta=7.84-7.73(\mathrm{~m}, 3 \mathrm{H}), 7.67(\mathrm{~d}, J=1.7 \mathrm{~Hz}, 1 \mathrm{H}), 7.54(\mathrm{dd}, J=8.6$, $1.7 \mathrm{~Hz}, 1 \mathrm{H}), 7.50-7.33(\mathrm{~m}, 7 \mathrm{H}), 6.68(\mathrm{~d}, J=15.7 \mathrm{~Hz}, 1 \mathrm{H}), 6.30(\mathrm{dt}, J=15.7,7.1 \mathrm{~Hz}, 1 \mathrm{H}), 4.12$ $(\mathrm{dd}, J=8.6,6.7 \mathrm{~Hz}, 1 \mathrm{H}), 3.31-3.07(\mathrm{~m}, 1 \mathrm{H}), 2.96-2.78(\mathrm{~m}, 1 \mathrm{H})$.

${ }^{13} \mathrm{C}$ NMR (101 MHz, $\left.\mathrm{CDCl}_{3}\right): \delta=169.66,136.89,134.59,133.71,133.41,133.06,129.18$, $128.31,128.23,128.08,127.78,126.39,126.15,126.05,125.94,123.56,77.48,77.36,77.16$, 76.84, 51.50, 37.07 .

${ }^{19} \mathrm{~F}$ NMR (376 MHz, $\left.\mathrm{CDCl}_{3}\right): \delta=-149.85--154.35(\mathrm{~m}),-157.93(\mathrm{t}, J=21.7 \mathrm{~Hz}),-162.31(\mathrm{td}, J$ $=22.3,5.0 \mathrm{~Hz})$.

HRMS (CI): $m / z$ calcd for $[\mathrm{M}]^{+} \mathrm{C}_{27} \mathrm{H}_{19} \mathrm{~F}_{5} \mathrm{O}: 468.11$. Found: 468.11.

HPLC analysis using a chiral column (Chiralpak IA $3 \mu$ column, $22{ }^{\circ} \mathrm{C}, 0.5 \mathrm{~mL} / \mathrm{min}, 400: 1$ Hexane:Isopropanol, $210 \mathrm{~nm}, \mathrm{t}_{\text {minor }}=23.4 \mathrm{~min}, \mathrm{t}_{\text {major }}=26.2 \mathrm{~min}$ ).

Pentafluorophenyl (E)-2-phenyl-5-(thiophen-3-yl)pent-4-enoate (24): Prepared according to

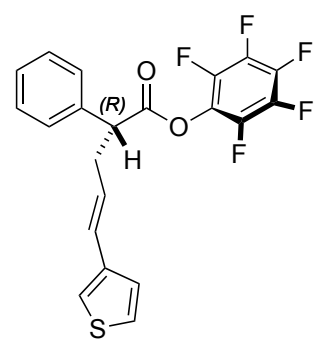

General Procedure C. The title compound 24 was obtained (40 mg, 0.095 mmol, 95\%) as a white solid following purification by column chromatography [ $\mathrm{SiO}_{2}$, Petroleum Ether-EtOAc, 400:1]. The enantiomeric excess $(98 \%$ ee) was determined by chiral HPLC in comparison with the racemate (see below).

M.p. (pentane-EtOAc, 400:1; plates) $=96-98{ }^{\circ} \mathrm{C}$

$[\alpha]_{\mathrm{D}}^{23}-19\left(\mathrm{c}=0.2, \mathrm{CHCl}_{3}\right)$.

IR (film): 1004, 1105, 1525, $1780 \mathrm{~cm}^{-1}$.

${ }^{1} \mathrm{H}$ NMR $\left(400 \mathrm{MHz}, \mathrm{CDCl}_{3}\right): \delta=7.40(\mathrm{~d}, J=4.3 \mathrm{~Hz}, 4 \mathrm{H}), 7.39-7.29(\mathrm{~m}, 1 \mathrm{H}), 7.28-7.21(\mathrm{~m}$, $1 \mathrm{H}), 7.14(\mathrm{dd}, J=5.1,1.3 \mathrm{~Hz}, 1 \mathrm{H}), 7.12-7.05(\mathrm{~m}, 1 \mathrm{H}), 6.52(\mathrm{~d}, J=15.8 \mathrm{~Hz}, 1 \mathrm{H}), 6.02(\mathrm{dt}, J=$ $15.8,7.1 \mathrm{~Hz}, 1 \mathrm{H}), 4.05$ (dd, $J=8.7,6.6 \mathrm{~Hz}, 1 \mathrm{H}), 3.14-2.98(\mathrm{~m}, 1 \mathrm{H}), 2.85-2.67(\mathrm{~m}, 1 \mathrm{H})$.

${ }^{13} \mathrm{C}$ NMR (101 MHz, $\left.\mathrm{CDCl}_{3}\right): \delta=169.64,139.75,136.88,129.16,128.21,128.04,127.47$, $126.12,125.53,125.03,121.79,77.48,77.36,77.16,76.84,51.51,36.87$.

${ }^{19} \mathrm{~F}$ NMR $\left(376 \mathrm{MHz}, \mathrm{CDCl}_{3}\right): \delta=-150.62--153.73(\mathrm{~m}),-157.95(\mathrm{t}, J=21.6 \mathrm{~Hz}),-161.20--$ 166.20 (m).

HRMS (CI): $m / z$ calcd for $[\mathrm{M}]^{+} \mathrm{C}_{17} \mathrm{H}_{11} \mathrm{~F}_{5} \mathrm{O}_{2}$ : 424.06. Found: 424.06.

HPLC analysis using a chiral column (Chiralpak IA $3 \mu$ column, $22{ }^{\circ} \mathrm{C}, 0.5 \mathrm{~mL} / \mathrm{min}, 400: 1$ Hexane:Isopropanol, $210 \mathrm{~nm}, \mathrm{t}_{\text {minor }}=11.319 \mathrm{~min}, \mathrm{t}_{\text {major }}=11.759 \mathrm{~min}$ ). 


\section{Large-scale preparations of 6 and 18.}

For large scale reactions lower catalyst loadings were employed (10 mol\% and 2 mol\%; for amine and Pd catalysts, respectively). These conditions were just as efficient although required $a$ reaction time of $12 \mathrm{~h}$.

(Pentafluorophenyl)2-phenylpent-4-enoate (6): An oven-dried round bottom flask containing a<smiles>C=CC[C@H](C(=O)Oc1c(F)c(F)c(F)c(F)c1F)c1ccccc1</smiles>
magnetic stirring bar was charged with XantphosPd (420mg, $0.44 \mathrm{mmol}, 2$ mol\%) and ester $4(6.04 \mathrm{~g}, 20.0 \mathrm{mmol}, 1.0$ equiv.) and evacuated and backfilled with nitrogen $(3 \times)$. Anhydrous THF $(100 \mathrm{~mL})$ was added via cannula followed sequentially by $i \operatorname{Pr}_{2} \mathrm{NEt}$ (3.6 mL, $22.0 \mathrm{mmol}, 1.1$ equiv.), (+)-BTM (509 mg, $2.0 \mathrm{mmol}, 10 \mathrm{~mol} \%)$ and allyl mesylate $\mathbf{S 1 8}(2.50 \mathrm{~mL}$, 22.0 mmol, 1.1 equiv.). The resulting solution was stirred at room temperature for $12 \mathrm{~h}$ whereupon TLC indicated complete consumption of 4 . Pentane $(30 \mathrm{~mL})$ was added and the suspension filtered through activated, acidic $\mathrm{Al}_{2} \mathrm{O}_{3}$ (the flask and $\mathrm{Al}_{2} \mathrm{O}_{3}$ were washed with $\left.3 \times \mathrm{Et}_{2} \mathrm{O}\right)$. The combined filtrates were dried $\left(\mathrm{Mg}_{2} \mathrm{SO}_{4}\right)$ and concentrated under reduced pressure. The residue was purified by column chromatography $\left[\mathrm{SiO}_{2}\right.$, Petroleum EtherEtOAc, 400:1] gave the title compound $6(6.29 \mathrm{~g}, 18.4 \mathrm{mmol}, 92 \%)$ as a colorless oil. The enantiomeric excess $(92 \%$ ee) was determined by chiral HPLC comparison with the racemate (as before, see page S15 for details).

Pentafluorophenyl (E)-2,5-diphenylpent-4-enoate (18): An oven-dried round bottom flask

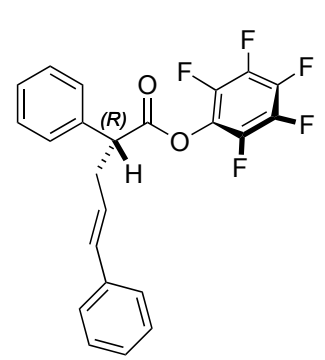

containing a magnetic stirring bar was charged with XantphosPd (420 mg, $0.44 \mathrm{mmol}, 2 \mathrm{~mol} \%)$ and ester 4 (6.04 g, $20.0 \mathrm{mmol}, 1.0$ equiv.) and evacuated and backfilled with nitrogen $(3 \times)$. Anhydrous THF $(100 \mathrm{~mL})$ was added via cannula followed sequentially by $i \operatorname{Pr}_{2} \mathrm{NEt}(3.6 \mathrm{~mL}, 22.0 \mathrm{mmol}$, 1.1 equiv.), (+)-BTM (509 $\mathrm{mg}, 2.0 \mathrm{mmol}, 10 \mathrm{~mol} \%$ ) and cinnamyl tertbutylcarbonate $\mathbf{S 1 8}$ (40.5 mL, $22.0 \mathrm{mmol}, 1.1$ equiv.). The resulting solution was stirred at room temperature for $12 \mathrm{~h}$ whereupon TLC indicated complete consumption of 4 . Pentane $(30 \mathrm{~mL})$ was added and the suspension filtered through activated, acidic $\mathrm{Al}_{2} \mathrm{O}_{3}$ (the flask and $\mathrm{Al}_{2} \mathrm{O}_{3}$ were washed with $3 \times \mathrm{Et}_{2} \mathrm{O}$ ). The combined filtrates were dried $\left(\mathrm{Mg}_{2} \mathrm{SO}_{4}\right)$ and concentrated under reduced pressure. The residue was purified by column chromatography $\left[\mathrm{SiO}_{2}\right.$, Petroleum Ether-EtOAc, 400:1] gave the title compound 18 (7.93 g, $19.0 \mathrm{mmol}, 93 \%)$ as a white solid. The enantiomeric excess (93\% ee) was determined by chiral HPLC comparison with the racemate (as before, see page S22 for details). 
Scheme 2. Transformations of 6 and confirmation of absolute stereochemistry.

2-phenylpent-4-en-1-ol (25): An oven-dried $20 \mathrm{~mL}$ vial was charged with pentafluorophenyl
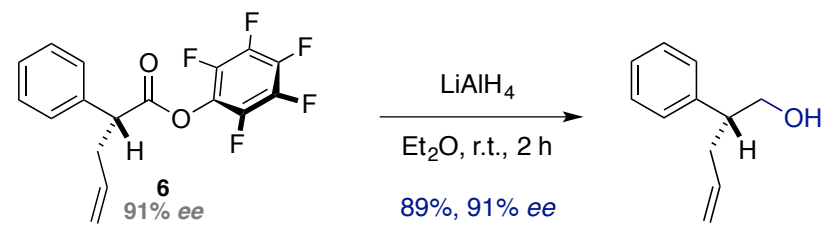
ester 6 (91\% ee, $100 \mathrm{mg}, 0.29 \mathrm{mmol}, 1.0$ equiv.) and evacuated and backfilled with nitrogen $(3 \times)$. Anhydrous $\mathrm{Et}_{2} \mathrm{O}(2 \mathrm{~mL})$ was added via syringe and the resulting solution cooled to $0{ }^{\circ} \mathrm{C}$ using an ice bath. A solution of $\mathrm{LiAlH}_{4}\left(1 \mathrm{M}\right.$ in $\mathrm{Et}_{2} \mathrm{O}, 0.29 \mathrm{~mL}, 0.32 \mathrm{mmol}$, 1.1 equiv.) was added dropwise via syringe and after complete addition the reaction was stirred at r.t. for $2 \mathrm{~h}$. Thereafter, aq. $\mathrm{HCl}(1 \mathrm{~N}, 5 \mathrm{~mL})$ was added dropwise (gas evolution!) and the layers separated. The aqueous phase was extracted with $\mathrm{Et}_{2} \mathrm{O}(3 \times)$. The combined organic exctracts were dried $\left(\mathrm{MgSO}_{4}\right)$ and concentrated under reduced pressure. The residue was purified by column chromatography $\left[\mathrm{SiO}_{2}\right.$, Pentane- $\mathrm{Et}_{2} \mathrm{O}, 10: 1$ ) to provide the title compound 25 (42 $\mathrm{mg}$, $0.26 \mathrm{mmol}, 89 \%$ yield) as a colorless oil. The enantiomeric excess ( $91 \%$ ee) was determined by chiral HPLC analysis in comparison with the racemate.

Spectroscopic data was consistent with the literature report for the racemic compound. ${ }^{16}$ $[\alpha]_{\mathrm{D}}^{23}=-15\left(\mathrm{c}=0.16, \mathrm{CHCl}_{3}\right) \cdot[\alpha]_{\mathrm{D}}^{20}=+15\left(\mathrm{c}=1.2, \mathrm{CHCl}_{3}\right)$ for the $(S)$-enantiomer. ${ }^{17}$

This confirms the absolute stereochemical configuration of 6 as $(R)$.

HPLC analysis using a chiral column (Chiralpak IA $3 \mu$ column, $22{ }^{\circ} \mathrm{C}, 1.0 \mathrm{~mL} / \mathrm{min}$, HexaneIsopropanol $\left.(99: 1), 210 \mathrm{~nm}, \mathrm{t}_{\text {minor }}=11.319 \mathrm{~min}, \mathrm{t}_{\text {major }}=14.018 \mathrm{~min}\right)$. HPLC chromatograms are presented below.

Methyl 2-phenylpent-4-enoate (26): An oven-dried 1 dram vial was charged with (sealed tube) pentafluorophenyl ester $6(91 \%$ ee, $50 \mathrm{mg}, 0.15$ mmol, 1.0 equiv.) and methanolic $\mathrm{HCl}(4 N, 0.15$ $\mathrm{mL})$ added. The vial was sealed and the reaction heated to $60{ }^{\circ} \mathrm{C}$ for $36 \mathrm{~h}$. Thereafter, the reaction was cooled to r.t. and concentrated under reduced pressure. The residue was dissolved in EtOAc and the resulting solution washed sequentially with $\mathrm{H}_{2} \mathrm{O}(3 \times)$ and sat. aq. Brine solution, dried $\left(\mathrm{Na}_{2} \mathrm{SO}_{4}\right)$ and concentrate under reduced pressure. The residue was purified by column chromatography $\left[\mathrm{SiO}_{2}\right.$, Pentane- $\left.\mathrm{Et} 2 \mathrm{O}, 10: 1\right)$ to provide the title compound 26 (16 $\mathrm{mg}, 0.084 \mathrm{mmol}, 56 \%$ yield) as a colorless oil. The enantiomeric excess $(91 \%$ ee) was determined by chiral HPLC analysis in comparison with the racemate.

Spectroscopic data was consistent with the literature report for the same compound. ${ }^{18}$

$$
[\alpha]_{\mathrm{D}}^{23}=-65\left(\mathrm{c}=0.22, \mathrm{CHCl}_{3}\right) . \text { Lit. }[\alpha]_{\mathrm{D}}^{25}=-89\left(\mathrm{c}=1.49, \mathrm{CHCl}_{3}\right) \text { for the }(R) \text {-enantiomer. }{ }^{18}
$$

This confirms the absolute stereochemical configuration of 6 as $(R)$. 
HPLC analysis using a chiral column (Chiralpak IB $3 \mu$ column, $22{ }^{\circ} \mathrm{C}, 0.5 \mathrm{~mL} / \mathrm{min}$, HexaneIsopropanol $\left.(400: 1), 210 \mathrm{~nm}, \mathrm{t}_{\text {major }}=13.3 \mathrm{~min}, \mathrm{t}_{\text {minor }}=14.7 \mathrm{~min}\right)$. HPLC chromatograms are presented below.

2-phenylpent-4-enoic acid (27): An oven-dried $20 \mathrm{~mL}$ vial was charged with pentafluorophenyl

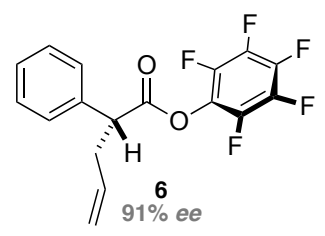

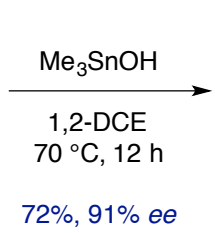

$72 \%, 91 \%$ ee

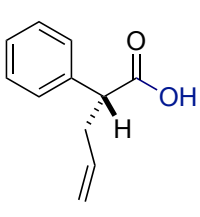

ester 27 (91\% ee, $50 \mathrm{mg}, 0.15 \mathrm{mmol}, 1.0$ equiv.) and trimethyltin hydroxide (264 mg, 1.46 mmol, 10 equiv.), and evacuated and backfilled with nitrogen $(3 \times)$. Anhydrous 1,2-dichloroethane $(2 \mathrm{~mL})$ was added via syringe, the vial was sealed and the reaction heated to $70{ }^{\circ} \mathrm{C}$ for $16 \mathrm{~h}$. Thereafter, the reaction was cooled to r.t. and concentrated under reduced pressure. The residue was dissolved in EtOAc $(4 \mathrm{~mL})$ and the resulting solution washed sequentially with $5 \%$ aq $\mathrm{HCl}(3 \times)$ and sat. aq. Brine solution, dried $\left(\mathrm{Na}_{2} \mathrm{SO}_{4}\right)$ and concentrate under reduced pressure. To the residue was added 1,2,4,5-tetramethylbenzene as an internal standard and the yield (72\%)* calculated by integration of the relevant signals in the ${ }^{1} \mathrm{H}-\mathrm{NMR}$ spectrum $(\delta=2.20 \mathrm{ppm}$, internal standard $=$ $1 \mathrm{H})$. The enantiomeric excess $(91 \%$ ee $)$ was determined by chiral HPLC analysis in comparison with the racemate.

* In a previous experiment, purification of the product carboxylic acid (from the residual pentafluorophenol) by column chromatography was unsuccessful due to the near-identical polarity of these components. As a result, the yield in this case was obtained through integration ( $\left.{ }^{l} H-N M R\right)$ of an internal standard (1,2,4,5-tetramethylbenzene) with signals corresponding to the carboxylic acid.

${ }^{1} \mathrm{H}$ NMR spectroscopic data was consistent with the literature report for the same compound. ${ }^{19}$

HPLC analysis using a chiral column (Chiralpak IB $3 \mu$ column, $22{ }^{\circ} \mathrm{C}, 1.0 \mathrm{~mL} / \mathrm{min}$, HexaneIsopropanol-Trifluoroacetic acid (98.9:1:0.1), $\left.210 \mathrm{~nm}, \mathrm{t}_{\text {major }}=12.1 \mathrm{~min}, \mathrm{t}_{\text {minor }}=14.0 \mathrm{~min}\right)$. HPLC chromatograms are presented below. Residual pentafluorophenol at $t_{r}=15.49$ min confirmed by co-injection. 
HPLC chromatograms for 25, 26 and 27.

Alcohol 25 Racemic Trace.

Chiralpak IA-3 Hexanes/Isopropanol 99:1; Flow rate= $1.0 \mathrm{ml} / \mathrm{min}$

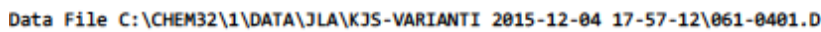

Sample Name: JLA-1-257-rac-A-99/1-1m]
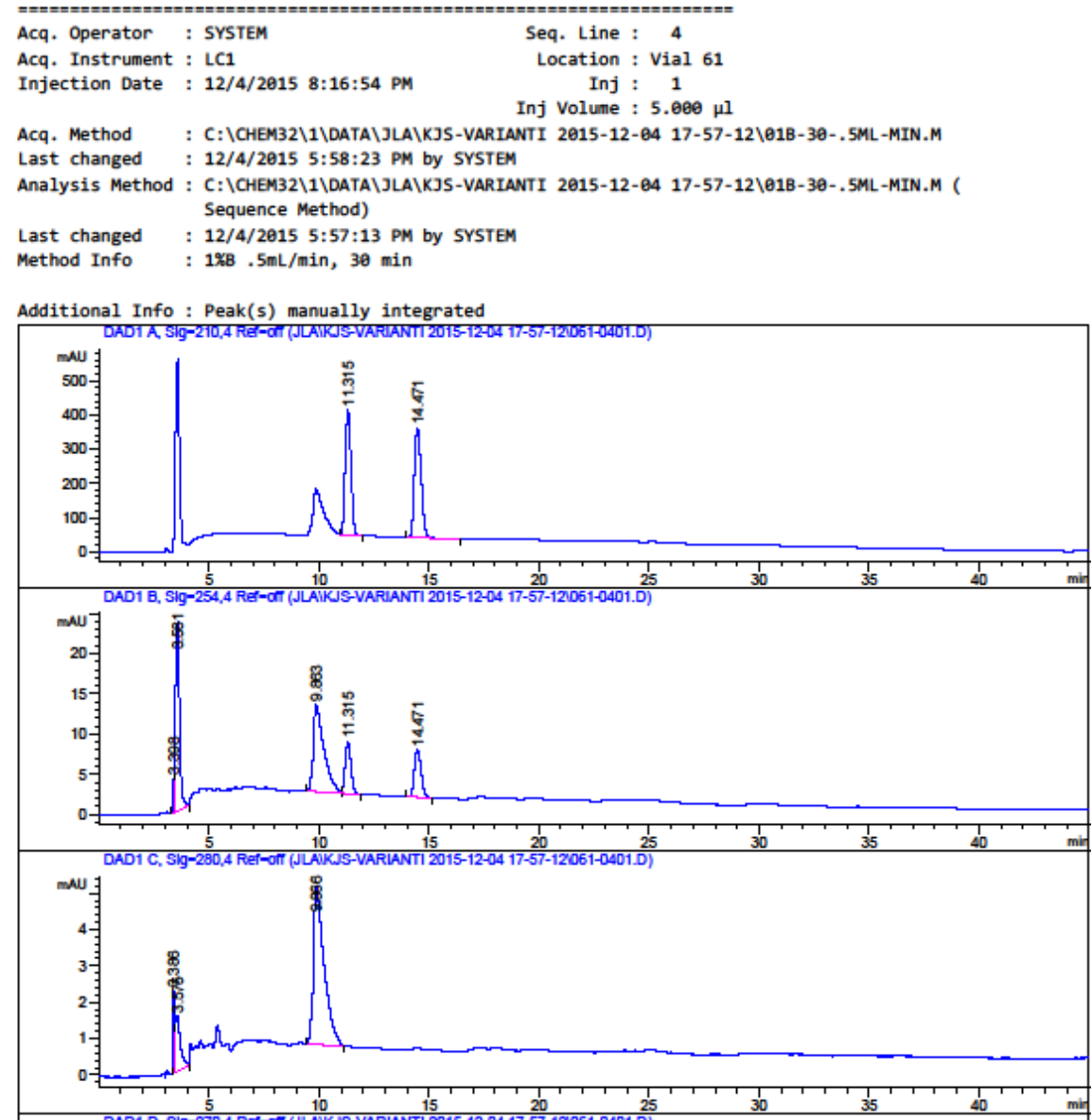
Alcohol 25 enantioenriched trace.

Chiralpak IA-3 Hexanes/Isopropanol 99:1; Flow rate= $1.0 \mathrm{ml} / \mathrm{min}$
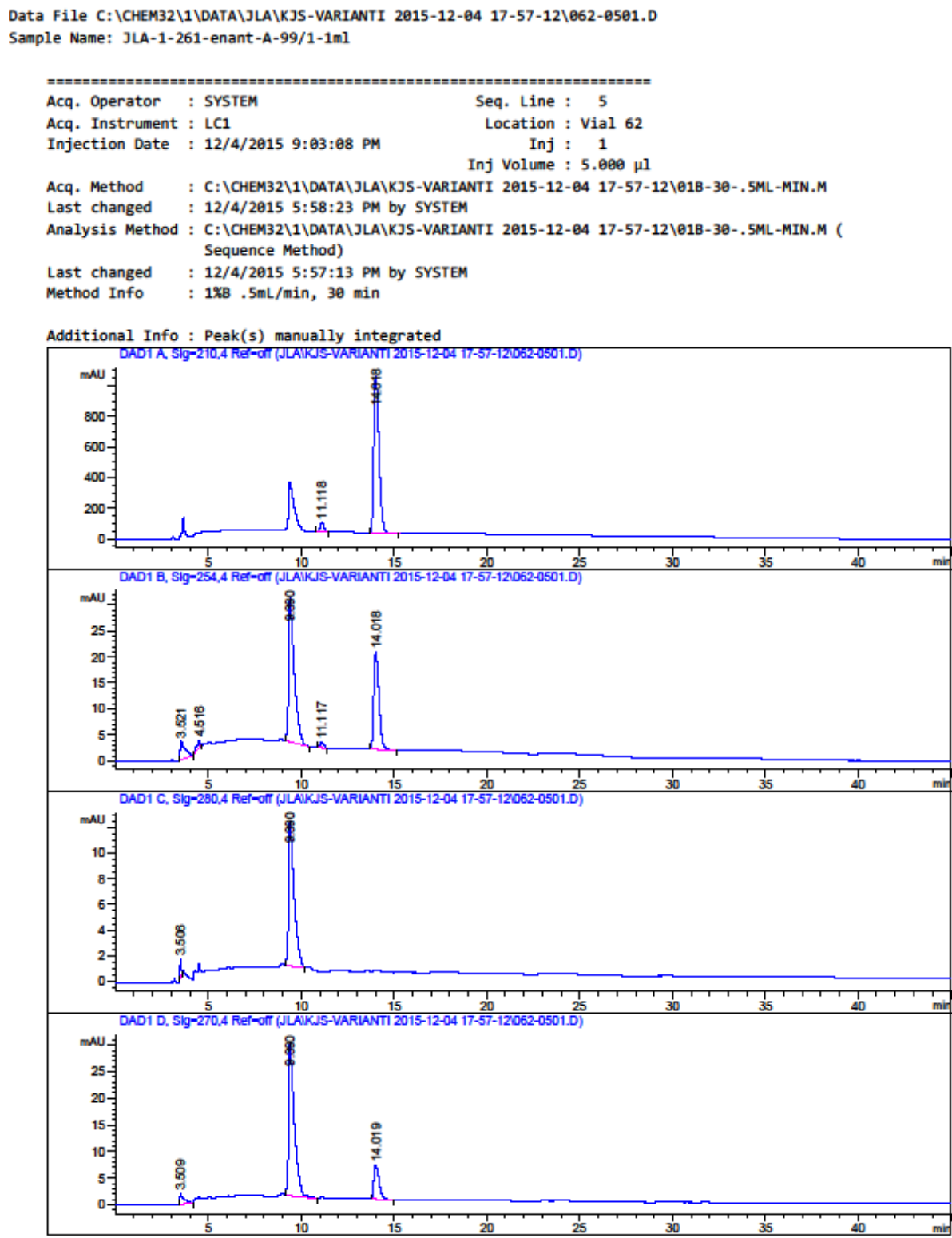
Methyl Ester 26 Racemic Trace.

Chiralpak IB Hexanes/Isopropanol 400:1; Flow rate $=0.5 \mathrm{ml} / \mathrm{min}$
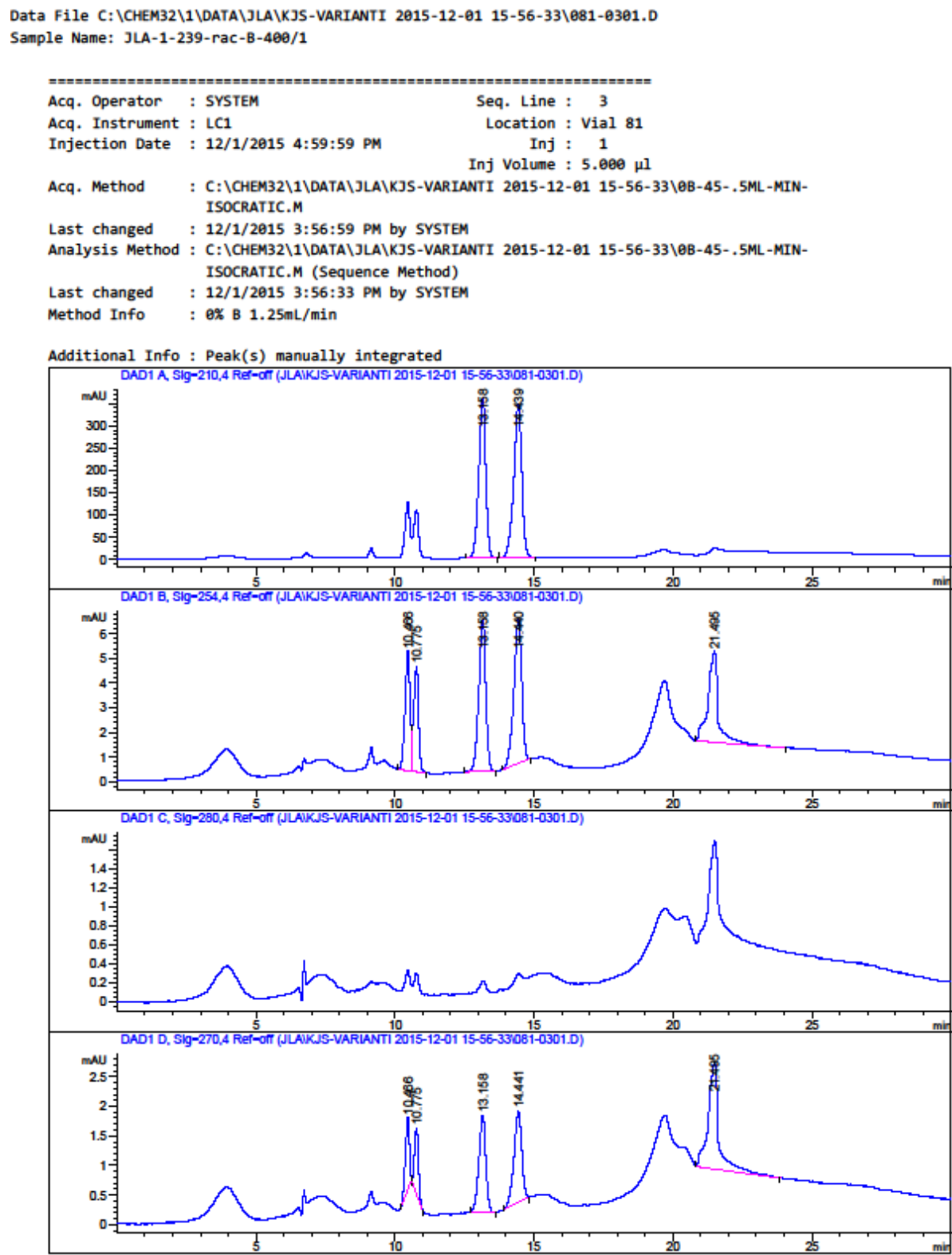

LC1 12/21/2015 3:46:09 PM SYSTEM

Page 1 of 3 
Methyl Ester 26 Enantioenriched Trace.

Chiralpak IB Hexanes/Isopropanol 400:1; Flow rate $=0.5 \mathrm{ml} / \mathrm{min}$

Data File C: \CHEM32\1\DATA\JLA\KJS-VARIANTI 2015-12-01 15-56-33\อ82-8401.D Sample Name: JLA-1-260-enant-B-460/1
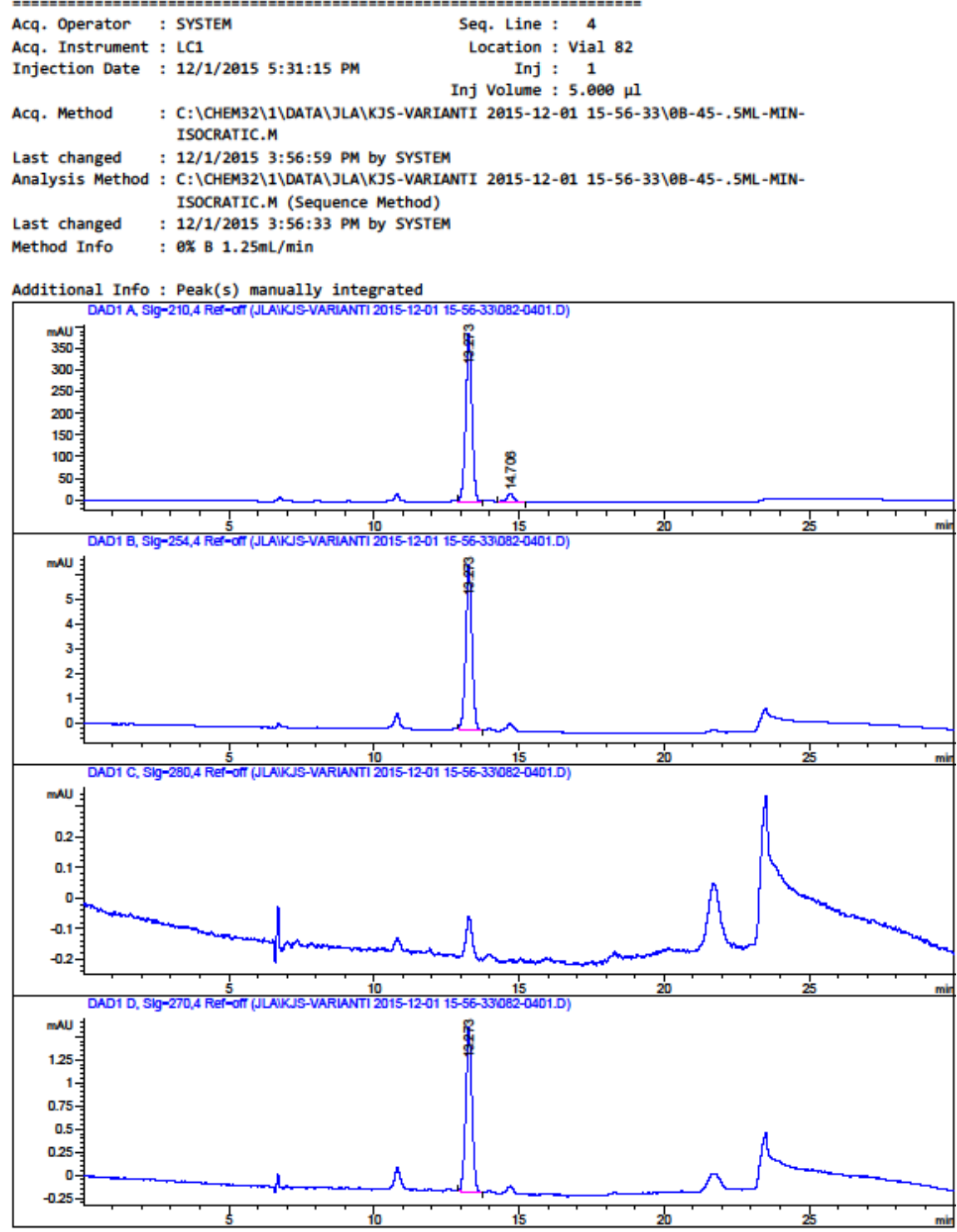

LC1 12/21/2015 3:45:35 PM SYSTEM

Page 1 of 3 
Carboxylic Acid 27 Racemic Trace.

Chiralpak IB, Hexanes/Isopropanol/trifluoroacetic acid 98.9:1;0.1 Flow rate=1.0 ml/min

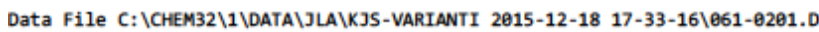

Sample Name: JLA-1-274-B-98/1/0.1-1m]

\begin{tabular}{|c|c|c|}
\hline Acq. Operator & : SYSTEM & Seq. Line : 2 \\
\hline Acq. Instrument & : LC1 & Location : Vial 61 \\
\hline Injection Date & : 12/18/2015 6:21:03 PM & $\begin{array}{rc}\text { Inj : } & 1 \\
\text { Inj Volume : } & 5 . \otimes 00\end{array}$ \\
\hline
\end{tabular}

Acq. Method : C: \CHEM32\1\DATA \JLA ISOCRATIC.M

Last changed : 12/18/2015 5:33:31 PM by SYSTEM

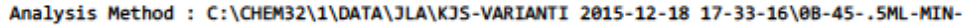

ISOCRATIC.M (Sequence Method)

Last changed : 12/18/2015 5:33:17 PM by SYSTEM

Method Info : $0 \%$ B $1.25 \mathrm{~mL} / \mathrm{min}$

Additional Info: Peak(s) manually integrated

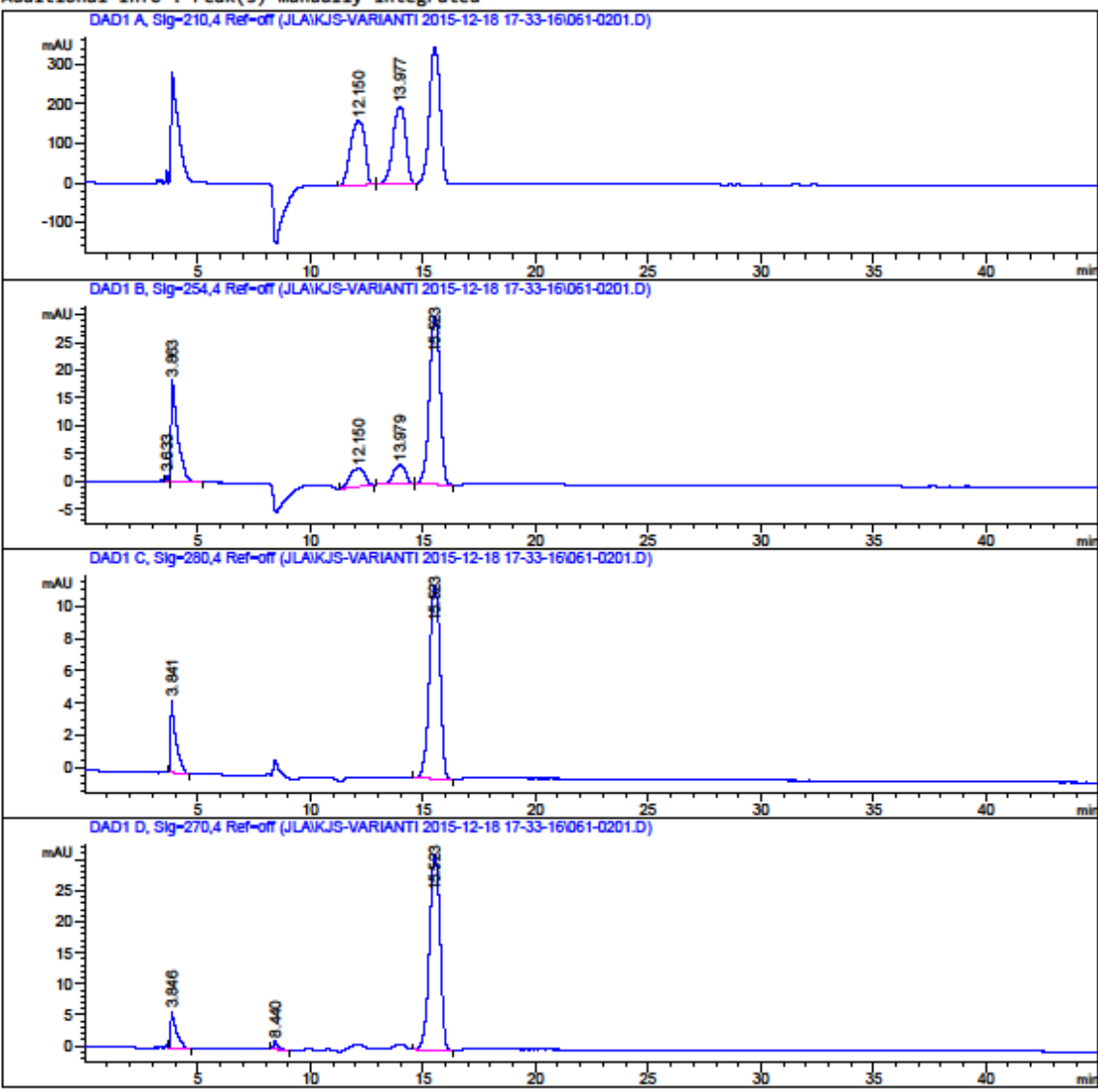

LC1 $12 / 21 / 2015$ 3:16:47 PM SYSTEM

Page 1 of 3 
Carboxylic Acid 27 Enantioenriched Trace.

Chiralpak IB, Hexanes/Isopropanol/trifluoroacetic acid 98.9:1;0.1 Flow rate=1.0 ml/min

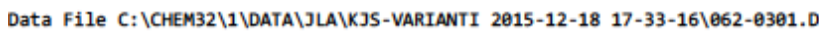
Sample Name: JLA-1-271-rt-B-98/1/0.1-1m1

$\begin{array}{ll}\text { Acq. Operator : SYSTEM } & \text { Seq. Line : } 3 \\ \text { Acq. Instrument : LC1 } & \text { Location : Vial } 62 \\ \text { Injection Date : } 12 / 18 / 2015 \text { 7:07:18 PM } & \text { Inj : } 1 \\ & \text { Inj Volume : 5.600 } \mu 1\end{array}$

Acq. Method : C: \CHEM32\1\DATA \JLA ISOCRATIC.M

Last changed : 12/18/2015 5:33:31 PM by SYSTEM

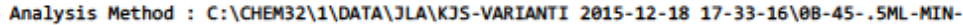

ISOCRATIC.M (Sequence Method)

Last changed : 12/18/2015 5:33:17 PM by SYSTEM

Method Info : $0 \%$ B $1.25 \mathrm{~mL} / \mathrm{min}$

Additional Info: Peak(s) manually integrated
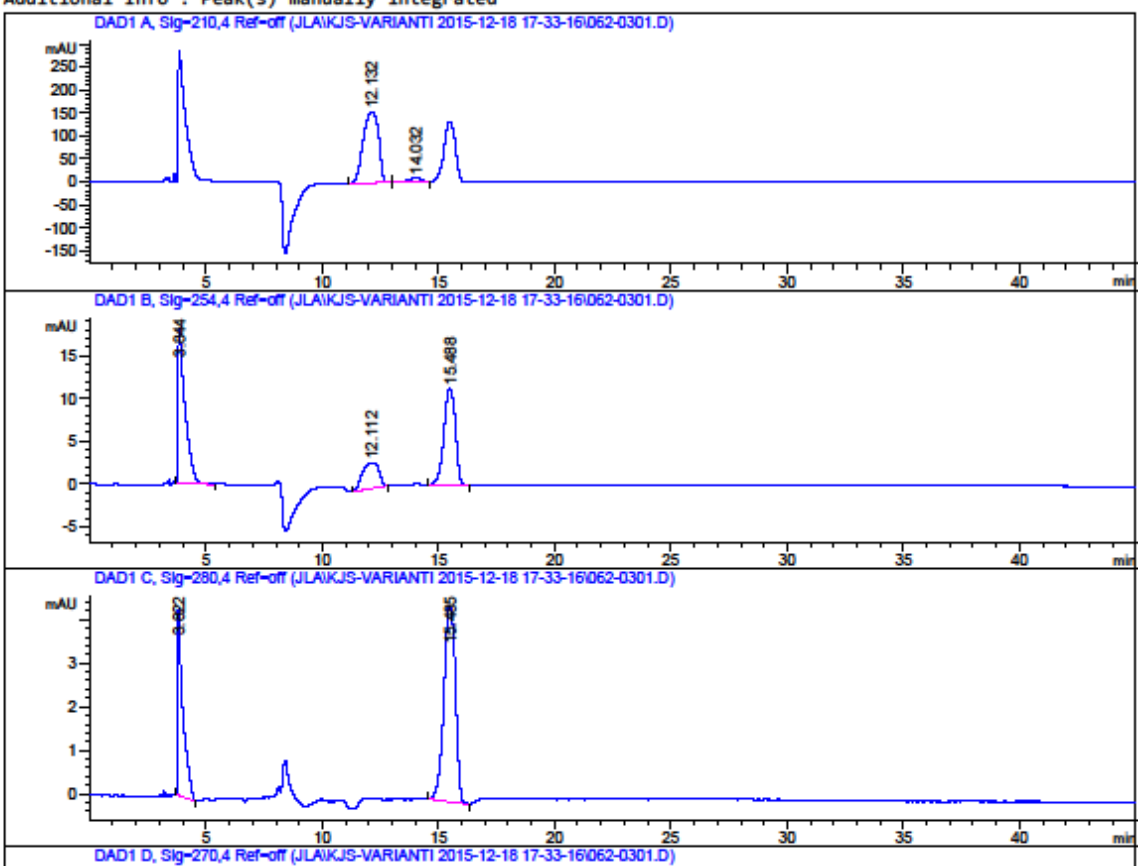

$$
\begin{gathered}
0 \\
10 \\
8 \\
6 \\
4 \\
2 \\
0
\end{gathered}
$$
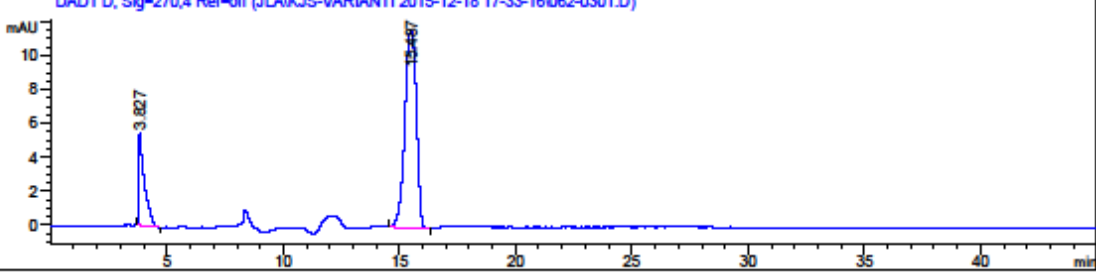

LC1 $12 / 21 / 2015$ 3:17:16 PM SYSTEM

Page 1 of 3 


\section{References}

1. D. D. Perrin, W. L. F. Amarego, Purification of Laboratory Chemicals, Pergamon Press, Oxford, Ed. 3, 1988.

2. Daniels, D. S. B.; Smith, S. R.; Lebl, T.; Shapland, P.; Smith, A. D. Synthesis 2015, 47, 34-41.

3. Bruno, N. C.; Tudge, M. T.; Buchwald, S. L. Chem. Sci. 2013, 4, 916-920.

4. Harnoy, A. J.; Rosenbaum, I.; Tirosh, E.; Ebenstein, Y.; Shaharabani, R.; Amir, R. J. Am. Chem. Soc. 2014, 136, 7531-7534.

5. Damakaci, F.; DeShong, P. J. Am. Chem. Soc. 2003, 125, 4408-4409.

6. Hatano, M.; Furuya, Y.; Shimmura, T.; Moriyama, K.; Kamiya, S.; Maki, T.; Ishihara, K. Org. Lett. 2011, 13, 426-429.

7. Guijarro, D.; Mancheño, B.; Yus, M. Tetrahedron 1992, 48, 4593-4600.

8. Hojo, M.; Sakuragi, R.; Okabe, S.; Hosomi, A. Chem. Commun. 2001, 4, 357-358.

9. $\quad$ Stead, D.; O’Brien, P.; Sanderson, A. J. Org. Lett. 2005, 7, 4459-4462.

10. Nakamura, A.; Hamasak, A.; Goto, S.; Utsonomiya, M.; Tokunaga, M. Adv. Synth. Cat. 2011, 353, 973-984.

11. Weix, D. J.; Marković, Ueda, M.; Hartwig, J. F. Org. Lett. 2009, 11, 2944-2947.

12. Trost, B M.; Fraisse, P. L.; Ball, Z. T. Angew. Chem., Int. Ed. 2002, 41, 1059-1061.

13. Stambasky, J.; Malkov, A.; Kocovsky, P. Collect. Czech. Chem. Commun. 2008, 73, $705-732$.

14. Houlihan, F.; Bouchard, F.; Fréchet, J. M. J.; Willson, C. G. Can. J. Chem. 1985, 63, 153-162.

15. Basel, Y.; Hassner, A. Synthesis 2001, 550-552.

16. Frantz, D. E.; Singleton, D. A. Org. Lett. 1999, 1, 485-486.

17. Wherritt, D. J. Ph.D. Thesis 2010, Ohio State University, p219.

18. Sarakinos G.; Corey, E. J. Org. Lett. 1999, 1, 1741-1744.

19. Stivala, C. E.; Zakarian, A. J. Am. Chem. Soc. 2011, 133, 11936-11939. 